\title{
THE FORGOTTEN TRADITION. HOW THE LOGICAL EMPIRICISTS MISSED THE PHILOSOPHICAL SIGNIFICANCE OF THE WORK OF RIEMANN, CHRISTOFFEL AND RICCI
}

\begin{abstract}
The paper attempts to show how the Logical Empiricists' interpretation of the relation between geometry and reality emerges from a "collision" of mathematical traditions. Considering Riemann's work as the initiator of a 19th century geometrical tradition, whose main protagonists were Helmholtz and Poincaré, the Logical Empiricists neglected the fact that Riemann's revolutionary insight flourished rather in a non-geometrical tradition dominated by the works of Christoffel and Ricci-Curbastro roughly in the same years. I will argue that in the attempt to draw the line Riemann-HelmholtzPoincaré-Einstein Logical Empiricists were led to argue that General Relativity raised mainly a problem of mathematical under-determination, i.e. the discovery that there are physical differences that cannot be expressed in the relevant mathematical structure of the theory. However, a historical reconstruction of the alternative line of development Riemann-Chritoffel-Ricci-Einstein shows on the contrary that the main philosophical issue raised by Einstein's theory was rather that of mathematical over-determination, i.e. the recognition of the presence of redundant mathematical differences that do not have any correspondence in physical reality.
\end{abstract}

In Logical Empiricists' philosophy of space and time, Einstein's conception of the relations between "geometry and experience" appears to be the heir of a 19th century philosophical and scientific tradition, whose main protagonists were Riemann, Helmholtz, and Poincaré. The result of such a tradition appeared to be at best framed in Reichenbach's celebrated theory of "equivalent descriptions". Riemann, Helmholtz and Poincaré discovered the "principle of the relativity of geometry", i.e. our freedom to chose among different metric geometries in as much as they can be transformed into one another by unique and continuous transformations, that is in as much as they are, in the Logical Empiricists' parlance, "topologically equivalent".

It is probably the merit of Michael Friedman to have convincingly shown that such a tradition simply never existed (Friedman, 1995). Helmholtz's and Poincaré's philosophy of geometry presupposes homogenous spaces, which can be covered by congruent tiles without gaps and overlappings. In such geometries there is a unique set of congruence relations, on which all observer can agree, or, more technically, a group of self-mappings with the properties of rigid motions can be defined. Riemann, on the contrary, left open the possibility of highly non-uniform spaces, where no group of motion can be defined and thus no unique conventional agreement can be made as to which tiles are congruent.

Logical Empiricists were of course aware of the elementary fact that there are no rigid bodies in spaces of variable curvature. However, by stripping Helmholtz and Poincaré philosophy of geometry from their group-theoretical implications (Friedman, 1995), they believed it was simply possible to shift the attention from "finite rigid bodies" to "infinitesimal rigid rods". As Roberto Torretti has shown, however, this strategy is hardly compatible with conventionalism. In a Riemannian manifold, an infinitesimal rod is considered rigid as long as it has an Euclidean behavior; one 
does not set by convention, which rods are rigid, but instead checks it under the hypothesis that the space is Euclidean in its smallest parts (Torretti, 1983).

In Riemannian geometry adopted in General Relativity, once a unit of measure as been arbitrarily fixed, the length of an the infinitesimal measure rod turns out to possess an absolute value, so that two intervals at a finite distance can be immediately compared. Einstein, it is true, refers, rather sporadically, to Helmholtz and Poincaré in his writing on the philosophy of geometry (Friedman, 2002). However, Einstein's reference should be rather understood in the context of the so called "measuring rod objection" against Hermann Weyl's attempt at unifying electricity and gravitation by dropping the length comparison "at-distance", rather then as a defense of conventionalism (Fogel, 2008, ch. 5).

Thus it has been abundantly shown that the Logical Empiricists' attempt to read Einstein's general theory as the heir of a geometrical tradition that starting with Riemann was developed into the epistemological works of Helmholtz and Poincaré was substantially flawed. In my opinion, however, an even more simple historical point has escaped recent historical literature. It is even more significant that the Logical Empiricists were not able to philosophically appreciate the fact that Riemann's work, roughly in the same years, was mainly developed in a nongeometrical tradition in the work of authors such as Elwin Bruno Christoffel and Gregorio Ricci-Curbastro, the father of the so called "absolute differential calculus" (our tensor calculus). Of course Einstein himself explicitly considered General Relativity precisely as the heir of this tradition, or, as he famously put it, "a real triumph of the method of the general differential calculus".

Riemann's work, considered from the point of view of Helmholtz's and Poincaré's philosophical reflections on geometry, appeared to the Logical Empiricist as concerned with the question of the choice among alternative physical geometries. Interpreted under the light of Christoffel's and Ricci-Curbastro's work, Riemann's main concern appears to be that of discerning the objective geometrical properties of the same physical geometry - those that are independent of the particular coordinate system we choose - from those properties that are a mere artifact of the coordinate system used.

As far I can see, the names of Christoffel and Ricci are not even mentioned by the Logical Empiricists. I would like to venture that this at least partially may have to do with the fact, that they never intervene in the philosophical debate on the foundation of geometry. In order to show that some quantity has geometrical substance and is not just an artifact of some arbitrary choice of coordinates, they merely invoked the abstract study of the law of transformation of the quadratic differential forms. However, it is only from this point of view that the mathematical apparatus of Riemannian geometry and most of all its role in General Relativity in the form of the requirement of "general covariance" - can be understood.

The aim of this paper is to show that the inadequacy (Friedman, 1983; Nerlich, 1994; Ryckman, 2007, 2008) of the Logical Empiricists' interpretation of General Relativity is in many respects the consequence of their failure to recognize the philosophical significance of this mathematical tradition. Logical Empiricists tried to interpret the role of Riemannian geometry in Einstein's General Relativity through the lenses of the epistemological problems raised by Helmholtz and 
Poincaré. Historically and systematically however Riemann's revolutionary approach became part of Einstein's theory of gravitation through the mediation of the analytical work of Christoffel and Ricci.

After a brief description of how the Riemann's insight was developed analytically (mainly, even if of course not only) by Christoffel and later by Ricci in the so called "absolute differential calculus" $(\$ 1)$ and then implemented in the General Theory of Relativity ( $(2)$,; the paper concentrates on Reichenbach's famous "conversion" to conventionalism and on the emergence of the standard logical empiricist interpretation of the relation between geometry and physics (§3).

The case of Reichenbach's appears particularly significant. Reichenbach's insistence on the "relativity of coordinates" in his first "Kantian" monograph (Reichenbach, 1920b) appears much more effective in hindsight than his later appeal to the "relativity of geometry" (Reichenbach, 1928). Reichenbach's deep knowledge of the mathematical apparatus of General Relativity shows that this change of position was not the consequence of a trivial "blunder", but the conscious pursuit of a philosophical program. The philosophical inadequacy of his reading emerges paradoxically by following the more expository/semi-technical parts his work, rather than concentrating on his philosophical interpretation $(\S 4)$.

Recent historically oriented philosophy of science has insisted on the importance of the 19th century debate on the foundation of geometry in order to understand the emergence of Logical Empiricism and, in particular, of its interpretation of the Theory of Relativity (Ryckman, 1992; Coffa, 1991; Friedman, 1999; Howard, 1994; Friedman, 2008, only to mention some titles). However, in my opinion, even recent well-informed and influential works (see of instance DiSalle, 2006) have not given sufficient attention to the implicit philosophical significance of the development of the Riemann's ideas in the work of Christoffel and Ricci. This investigation was therefore left exclusively to the history of mathematics (see for instance the classical Reich, 1994), rather than being considered a part of that "synthetic" history (Dickson and Domski, 2010) that eventually led to the emergence of modern philosophy of science.

The filling of this lacuna in the historical literature on Logical Empiricism could help to clarify a more general philosophical misunderstanding, which seems to characterize early philosophical interpretations of General Relativity. General Relativity, considered as the heir to the 19th century conventionalism of Helmholtz and Poincaré, seemed to raise the problem of mathematical under-determination; it shows the existence of mathematically equivalent, but different physical geometries, among which we can only make a pragmatic choice. Considered as the heir to the work of Riemann, Christoffel and Ricci, General Relativity appears mainly to raise a problem of mathematical over-determination; it shows that it is possible to represent the same physical geometry in different coordinate systems, using different mathematical functions. General Relativity shows the existence of a "redundancy" in the mathematical description, rather than "reducing" the mathematical structure which is physically relevant. 


\section{Riemann's “Äquivalenzproblem” and its Analytical Development in the Work of Christoffel and Ricci-Curbastro}

1.1. Riemann: from Habilitationsvortrag to the Commentatio Mathematica. On June 10 1854, before the Philosophical Faculty at Göttingen, Riemann delivered his celebrated Habilitationsvortrag, Ueber die Hypothesen, welche der Geometrie zu Grunde liegen. This short address will turn out to be one of the most influential (even if only partially understood) papers in the second half of the nineteenth century.

Riemann famously considered space as a special case of " $n$-dimensioned manifoldness" (Riemann 1854/1868, 138; tr. Riemann, 1873; cf. Scholz, 1982), expressed by means of " $n$ variables $x_{1}, x_{2}, x_{3}, \ldots, x_{n}$ " (Riemann, 1854/1868, 139; tr. 1873, 15). Inspired by Gauss's theory of curved surfaces (Gauss, 1827), Riemann assumed as an hypothesis (as the simplest among other possible alternatives) that space is distinguished from other "manifoldnesses" by the fact that the so called line element $d s$, "is the square root of an always positive integrable homogeneous function of the second order of the quantities $d x$, in which the coefficients are continuous functions of" the quantities $x "$, (Riemann 1854/1868, 140; tr. 1873, 15; cf. Libois, 1957; Scholz, 1992). As is well known, Riemann's abstract approach turned out to be extremely powerful, allowing an infinity of possible geometries. Different geometries correspond to different expressions for the line element endowed by different sets of coefficients.

The opposite, however, is not necessarily true. Riemann observed that every such expression can be transformed "into another similar one if we substitute for the $n$ independent variables functions of $n$ new independent variables" (Riemann $1854 / 1868,140 ;$ tr. 1873,16$), x_{1}, \ldots x_{n}$. The coefficients of the quadratic expression will depend on the variable used, but it is possible to show how the coefficients transform under a change of the independent variables in such a way as to make $d s^{2}$ remain unchanged.

However, as immediately Riemann made clear, "we cannot transform any expression into any other" (Riemann 1854/1868, 140; tr. 1873, 16). The fact that a sphere cannot be projected onto a plane without distortion can be expressed analytically by the fact that it is impossible to convert the quadratic differential form, which holds on a sphere by means of a mere transformation of the independent variables, to one "in which the square of the line-element is expressed as the sum of the squares of complete differentials", that is to one "in which the line-element may be reduced to the form $\sqrt{\sum d x^{2}}$ " (Riemann 1854/1868, 141, tr. 1873, 16); for more details Portnoy, 1982; Zund, 1983).

Different geometries are expressed analytically by different line elements, but the difference in the appearance of the line element does not necessary imply a geometrical difference. One of the main problems raised by Riemann's inquiry was that of discerning the geometrical properties that do not depend on a particular choice of the independent variables, from those that are a mere deceptive appearance introduced by the mathematical formalism.

Riemann's speech, which was supposed to be held in front of an audience of non-mathematicians, was intentionally scarce in the use of mathematical formulas. However, Riemann's somehow cryptic parlance becomes more familiar to the modern reader, if one considers the notation that he introduced in the so called 
Commentatio mathematica (Riemann, 1861/1876, tr. Farwell, 1990, 240-253) a paper submitted to the Paris Academy in 1861, to compete for a prize relating to the conduction of heat in homogeneous bodies with constant conductivity coefficients (the prize was not assigned), but that remained unknown until 1876, when Richard Dedekind - who has found it in Riemann's Nachlaß together with the Habilitationsvortrag - published it in the first edition of Riemann's work.

In order to address the question posed by the academy, Riemann had to bring a system of partial differential equations into its simplest form. The problem turned out to be equivalent to that of reducing a quadratic differential from $\sum a_{i, i^{\prime}} d x_{i} d x_{i^{\prime}}$ (where $a_{i, i^{\prime}}$ represent conductivity coefficients), into the form $\sum d x_{i}^{2}$ with constant coefficients, by a mere change of the independent variables $x_{i}$ (where $i=1,2,3$ ) (Riemann, 1861/1876, 392, tr. Farwell, 1990). ${ }^{1}$

Since it would be tedious to try various transformations of variables to establish the possibility of such a reduction, Riemann wanted to find a general criterion of transformability (see Farwell, 1990). For this purpose he introduced a four-index symbol $\left(\iota \iota^{\prime}, \iota^{\prime \prime} \iota^{\prime \prime \prime}\right)$ containing the first and and the second partial derivatives of the functions $a_{i, i^{\prime}}$ respect to the $x_{i}$. Then he showed that a quadratic differential form can be transformed in one with constant coefficients if the four-index symbol vanishes: $\left(\iota \iota^{\prime}, \iota^{\prime \prime} \iota^{\prime \prime \prime}\right)=0$ (Riemann, 1861/1876, 402, tr. Farwell, 1990). Riemann found a criterion for distinguishing between the case in which the non-constancy of the conductivity coefficients $a_{i, i^{\prime}}$ is a mere appearance of the mathematical description and the case in which it corresponds to a real physical difference, i.e. to a thermally non-homogeneous body.

Riemann hints, although vaguely, at a geometrical interpretation of this mathematical apparatus. The quadratic form $\sqrt{\sum_{\iota, \iota^{\prime}} b_{\iota, \iota^{\prime}} d s_{\iota} d s_{\iota^{\prime}}}$ can be regarded as the "line element in a more general space of $n$ dimensions extending beyond the bounds of our intuition" (Riemann, 1861/1876, 435, tr. Farwell, 1990, 240-253). A "flat space" can be represented by a quadratic differential form, whose coefficients are non-constant, such as polar coordinates or more complicated curvilinear coordinates. This difference however does not have any geometrical meaning; it is always possible to find a transformation of variables with which the form $\sum b_{\iota, \iota^{\prime}} d s_{\iota} d s_{\iota^{\prime}}$ can be transformed into one constant coefficients $\sum_{\iota} d_{\iota}^{2}$ (Riemann, 1861/1876, 435, tr. Farwell, 1990, 240-253). In a non-flat space, on the contrary, such a transformation cannot be found.

The four-index symbol furnishes a precise mathematical criterion: when it vanishes the non-constancy of the coefficients is merely an artifact of the system of variables used, if not, the non-constancy has, so to say, geometrical substance. Geometrically the four-index symbol corresponds then to the "measure of the curvature of this surface at the point $\left(s_{1}, s_{2}, \ldots, s_{n}\right)$ " (Riemann, 1861/1876, 435, tr. Farwell, 1990). In his 1854 lecture Riemann had famously shown that this is not necessarily constant, opening the possibility of space with a variable curvature. Spaces of constant curvature are merely a special case, where "independence of bodies from position" is assured (Riemann, 1854/1868, 149; tr. 1873, 36).

The Commentatio seems however to make clear that Riemann's main concern was capable of being expressed in a purely analytical way - i.e. independently

\footnotetext{
${ }^{1}$ For sake of historical accuracy along the paper we will try to remain faithful to the original notations used by the various authors considered.
} 
from its possible physical or geometrical interpretation - as the problem of the equivalence of differential quadratic forms. This problem, which is best known as the Äquivalenzproblem, exerted a profound influence on the later development of mathematics and physics, as the development of absolute differential calculus (Tonolo, 1961; Struik, 1993) as its implementation in Einstein's theory of relativity shows. In my opinion, however, this was not fully appreciated in the philosophical debate raised by the appearance of Einstein's theory of gravitation. The work of Riemann, on the contrary, was read uncritically under the light of Helmholtz's and Poincaré's philosophy of geometry, who, as we shall see, were concerned by quite different philosophical questions.

\subsection{A Very Brief History of the Emergence of the Absolute Differential} Calculus: Christoffel, Ricci and Levi-Civita. Riemann's Habilitationsvortrag was discovered in the late 1860s by Richard Dedekind, who had been entrusted with Riemann's Nachlaß, and published in the "Abhandlungen der Königlichen Gesellschaft der Wissenschaften zu Göttingen," vol. 13, 1868 (Riemann, 1854/1868). Immediately afterwords, Helmholtz, who had known of the existence of Riemann's paper in 1868 from Ernst Schering (Koenigsberger, 1906, 139), published his famous Über die Tatsachen, die der Geometrie zu Grunde liegen, which appeared a little later in the "Göttinger gelehrte Nachrichten," vol. 15, 1868 (Helmholtz, 1868).

Helmholtz famously derived Riemann's hypothesis that metric relations are given by a quadratic differential form (and not, for instance, by a quartic differential form) from the fact that there are rigid bodies, whose translations and rotations - expressed analytically by a set of differential equations - necessarily leaves a quadratic differential form unchanged. As is well known, Helmholtz's approach based on the notion of rigid body was enormously successful in the history of the philosophy of geometry.

As early as 1870, Helmholtz himself discussed the epistemological implications in the less technical and widely read talk Über den Ursprung und die Bedeutung der geometrischen Axiome (Helmholtz, 1870/1883) and in other philosophical papers that followed (Helmholtz, 1878, 1879). In 1886 Sophus Lie (Lie, 1886), stimulated by Felix Klein, reinterpreted and corrected Helmholtz's result on the basis of his theory of continuous groups. In 1887 Henri Poincaré (who already insisted on the group-theoretical approach on rigid motions in the early 1880s; Poincaré, 1882, §2), referred to Lie's results in his fist paper on the foundations of geometry (Poincaré, 1887, 214). At the end of the paper, Poincaré hints to the "celebrated Memoire of Riemann", in which every geometry is characterized "through the expression of the arc element as a function of the coordinates" (Poincaré, 1887, 214). However, he discarded it as geometrically irrelevant, because it allows for spaces which exclude "the existence of a group of motion which does not alter distances" (Poincaré, 1887, 214, see also Poincaré, 1891, 773).

In general, Riemann's speculations about variably curved spaces (with the notorious exception of Clifford, 1876) were either ignored or dismissed (Hawkins, 1980, 2000). Rather, Riemann's paper triggered developments in the non-geometrical branch of mathematics concerned with the study of quadratic differential forms. Dedekind had mentioned Riemann's unpublished Habilitationsvortrag to Erwin Bruno Christoffel, which in 1862 filled Dedekind's post at ETH in Zurich (Butzer, 1981). 
In the last paragraph of the paper published in 1869 on "Journal für die reine und angewandte Mathematik" (the celebrated Crelle's Journal), Über die Transformation der homogenen Differentialausdrücke zweiten Grades (Christoffel, 1869), Christoffel in fact referred briefly to "an essay [Abhandlung] in Riemann's Nachlass" (Christoffel, 1869). Christoffel's paper addressed the equivalence problem for two quadratic differential forms in the most general way, without focusing on special case the reducibility to an expression with constant coefficients (Ehlers, 1981).

Christoffel wanted to establish which "conditions are necessary [erforderlich]" (Christoffel, 1869), for transforming a quadratic differential form $F=\sum \omega_{i k} \vartheta x_{i} \vartheta x_{k}$ into another such form $F^{\prime}=\sum \omega_{i k}^{\prime} \vartheta x_{i}^{\prime} \vartheta x_{k}^{\prime}$ by means of a smooth, invertible substitution of the independent variables. In order to answer this question, Christoffel was led "to a better overview [zur besseren Übersicht]" (Christoffel, 1869) to express the recurrent combination of the $\omega_{i k}$ and their first partial derivatives $\frac{\partial \omega_{g k}}{\partial x_{h}}$ through two kinds of three-index symbols $\left[\begin{array}{c}g h \\ k\end{array}\right]$ and $\left\{\begin{array}{l}i l \\ r\end{array}\right\}=\sum \frac{E_{r k}}{E}\left[\begin{array}{c}g h \\ k\end{array}\right] 2$ (the now famous Christoffel symbols respectively of first and second kind; Reich, 1994; Herbert, 1991).

The purely "algebraic" criterion for the equivalence of two differential forms is then obtained by introducing a four-index symbol $(g h k i)$, that can be constructed from the "Christoffel symbols" and their derivatives (that is from the first and second partial derivatives of the $\omega_{i k}$ ). It corresponds to Riemann's four-index symbol in the Commentatio (which Christoffel could not know). Two quadratic form can be transformed into one another only if (locally) $(g h k i)=(g h k i)^{\prime}$.

No reference to the geometrical concept of "curvature" can be found in Christoffel's paper, which follows a purely algorithmic approach. This attitude was taken up by Gregorio Ricci-Curbastro, who in six papers published between 1883-1888 was able to develop systematically Chritoffel's solution of the Äquivalenzproblem into a new calculus (Dell'Aglio, 1996; Bottazzini, 1999). In his first paper on the argument, Principii di una teoria delle forme differenziali quadratiche (Ricci-Curbastro, 1883), Ricci recognized his debts to Riemann (Ricci could now refer to Riemann's Commentatio), Rudolf Lipschitz (Lipschitz, 1869) and most of all Christoffel.

As Christoffel, Ricci did not want to give a contribution to geometry. According to Ricci "mathematicians have usually considered quadratic differential forms . . a as representing line elements of $n$-dimensional spaces" (Ricci-Curbastro, 1883, 140). This, however, has often led to confusion. Ricci explicitly emphasized that his own investigations are based "on purely analytical concepts", letting apart "the rather vacuous [oziose] discussions about the existence of spaces on more than three dimensions" (Ricci-Curbastro, 1883, 140).

Ricci's aim was a purely abstract theory of differential invariants, a calculus of quadratic differential forms, such as " $\varphi=\sum_{r s} a_{r s} d x_{r} d x_{s}$ where $a_{r s}$ are functions of $x_{1}, x_{2}, . ., x_{n}$." (Ricci-Curbastro, 1886, 3). The problem was then to establish the laws according to which the coefficients would, by a change of the independent variables when "one substitutes the variables $x_{1}, x_{2}, . ., x_{n}$ with the variables $u_{1}, u_{2}, . ., u_{n}$ " (Ricci-Curbastro, 1886, 4) (which are smooth functions of the first ones) under the condition that $\varphi=\varphi^{\prime}$.

\footnotetext{
$2 \frac{E_{r k}}{E}$ corresponds to the inverse matrix of $\omega_{i k}$
} 
Ricci showed that the coefficients $a_{r s}$ transform according to certain rules into the new coefficients $\left(a_{p q}\right)$, so that the new form is called "covariant" respect to the first; the reciprocal form $a^{(r s)}$ transformed "contravariantly" into $a^{(p q)}$ (RicciCurbastro, 1889, 113). Hence in Ricci's parlance $a_{r s}$ and $a^{(r s)}$ form respectively a covariant and contravariant "system" of second rank (because of the two indeces).

To establish the conditions of tranformability, Ricci followed Christoffel's path introducing the three index-symbol $a_{r s, i}$ (for the Christoffel symbols of first kind), and the four index-symbol $a_{h i, j k}$ which he later called "the system of Riemann" (Levi-Civita and Ricci-Curbastro, 1900, 142). However, Ricci, starting from his 1887 paper Delle derivazioni covarianti e controvarianti e del loro uso nella analisi applicata (Ricci-Curbastro, 1888) interpreted Christoffel's algorithms as a differentiation of a more general kind that he labeled as "covariant (contravariant) differentiation". (Ricci-Curbastro, 1888, 4). With the repeated application of the covariant differentiation, starting from a covariant (or contravariant) primitive system, others can be obtained (Ricci-Curbastro, 1888, §2).

This was the necessary step which allowed Ricci to traduce Christoffel's still unsystematic approach into a new "calculus". In 1893 Ricci called it for the first time "absolute differential calculus," where the word "absolute" expresses the fact the calculus can be applied "independently of the choice of the independent variables" and requires "that the latter are completely general and arbitrary" (RicciCurbastro, 1893). Ricci made his results known outside of Italy with a summary published in French in Georges Darboux's "Bulletin des Sciences Mathématiques" in 1892 (Ricci-Curbastro, 1892). Only in 1901, with the assistance of his student Tullio Levi-Civita, did he publish a memoir in French, which can be considered the manifesto of Ricci's calculus, "Méthodes de calcul différentiel absolu et leurs applications" in Klein's journal, "Mathematische Annalen" in 1900 (Levi-Civita and Ricci-Curbastro, 1900),

\section{2. "A Real Triumph of the Method of the General Differential Calculus": Einstein's General Relativity}

Ricci's calculus apparently failed in finding his audience among differential geometers, who were probably its main target (Roth, 1942, 266; Reich, 1994, 77, but see Bottazzini, 1999). Ricci's algorithms appeared incapable of providing any substantially new geometrical results that could not have been reached through a more traditional approach. This was what Luigi Bianchi, author of celebrated handbook on differential geometry (Bianchi, 1894), meant when, reviewing Ricci's work for the royal prize of the Accademia dei Lincei, characterized it as "useful but not indispensable" (Bianchi, 1902, 149; on the relations Bianchi-Ricci see Toscano, 2001).

It is usually argued that only General Relativity eventually did justice to Ricci's work. Einstein's progressive appropriation of the work of Riemann, Christoffel, Ricci, and Levi-Civita has been of course discussed several times in historical literature (see for instance: Earman and Glymour, 1978; Stachel, 1980/2002; Norton, 1984; Janssen and Renn, 2007; Pais, 1982, 212ff.)). We will here only give a very superficial presentation in order to emphasize some elements that will be relevant to understanding the subsequent philosophical discussion.

2.1. Einstein, Grossmann and the Absolute Differential Calculus. In 1907 Einstein put forward the Principle of Equivalence as an extension of the Relativity 
Principle to uniformly accelerated systems (Einstein, 1907). After having embraced Minkowski's geometrical interpretation of special relativity, in 1912 Einstein, as he later recalled (Einstein, 1922), had grasped the "decisive idea": the analogy between his work on extending the principle of relativity to accelerated motion with Gauss's theory of surfaces, "without being aware at that time of the work of Riemann, Ricci, and Levi-Civita" (Einstein, 1922).

It was famously Marcel Grossmann, who in August 1912, introduced Einstein to this mathematical tradition: "never before in my life" - as Einstein wrote in a famous letter to Arnold Sommerfeld in the October of same year — "have I gained enormous respect for mathematics, whose more subtle parts I have considered until now, in my ignorance, as pure luxury" (CPAE, Vol. 5, Doc. 421)

Einstein and Grossmann jointly published the Entwurf einer verallgemeinerten Relativitätsheorie und einer Theorie der Gravitation (Einstein and Grossmann, 1913). Einstein's "Entwurf" theory (just as his final general theory of relativity) is built around a quadratic differential form $d s^{2}=\sum g_{\mu \nu} d x_{\mu} d x_{\nu}$ that assumes the name of "metric tensor" or "fundamental tensor" (which corresponds to Ricci's "covariant system of second rank" Einstein and Grossmann, 1913, 25, n.). The physical novelty consisted of course in the fact that the coefficients $g_{\mu \nu}$ of the quadratic differential form represent the behavior of measuring-rods and clocks with reference to the coordinate system, as well as the potentials of the gravitational field. The geodesic trajectories of particles can be considered as the solutions to the variational problem $\delta\left\{\int d s\right\}=0$.

Grossman/Einstein's problem can be formulated as follows: "What differential equations permit us to determine the quantities $g_{\mu \nu}$ i.e., the gravitational field?" (Einstein and Grossmann, 1913, 11) Einstein's strategy was famously to seek for a "generally covariant" analogon of the Poisson's equation $\frac{\partial^{2} \varphi}{\partial x^{2}}+\frac{\partial^{2} \varphi}{\partial y^{2}}+\frac{\partial^{2} \varphi}{\partial z^{2}}=4 \pi k \rho$. The ten potentials $g_{\mu \nu}=g_{\nu \mu}$ play the role of the single potential $\varphi$, whereas the density $\rho$ corresponds to the ten components of a second rank tensor $\Theta_{\mu \nu}$, the so-called stress-energy tensor. The problem was to find a second rank tensor $\Gamma_{\mu \nu}$, constructed only from the $g_{\mu \nu}$ and their first and second derivatives with respect to the coordinates (just like the Poisson's equation equation involves the second derivative of the potential), which should then play the role of the gravitational tensor: Thus the field equations "would likely have the form $\kappa \cdot \Theta_{\mu \nu}=\Gamma_{\mu \nu}$ where $\kappa$ is a constant" (Einstein and Grossmann, 1913, 11).

As the Zurich Notebook reveals, Grossman had immediately found a "tensor of fourth manifold" (Tensor vierter Mannigfalfigkeit) - $(i k, l m)$ using the four-indexsymbol notation - as the only tensor that can contains only the metric tensor and its first and second derivatives (Sauer, 2005). It turned out to be much more complicated to find the exact form of the "covariant differential tensor of second rank and second order" (Einstein and Grossmann, 1913, 36), obtained from the Riemann-Christoffel tensor by contraction (that is by setting unlike indices equal) that could play the role of the gravitational tensor (having the same valence and rank of the matter tensor).

Einstein and Grossman discarded the natural candidate, the so called RicciTensor (Maltese, 1991), since "it does not reduce to $\Delta \phi$ ", that is to the Newtonian limit (assumed erroneously as spatially flat), "in the case of weak static field" (Einstein and Grossmann, 1913, 337). In the subsequent years Einstein gave up general covariance for the equations of gravitational field. An argument, which came to be 
known as the "hole argument", even convinced Einstein that, as he wrote to Paul Ehrenfest in early 1914, "generally covariant field equations that determine the field completely from the matter tensor cannot exist at all" (CPAE, Doc. 512, 5, 563). ${ }^{3}$ In March of the same year Einstein even wrote to Michele Besso that "[t]he general theory of invariants only acted as a hindrance" (CPAE, 5, Doc. 514, 604).

In October 1914, Einstein, who in the meantime had moved to Berlin, presented a systematic exposition of the Entwurf theory to the Berlin Academy Die formale Grundlage der allgemeinen Relativitätstheorie (Einstein, 1914). §7, "Geodesic line or equations of the point motion", introduced a fundamental formal innovation, using the absolute differential calculus to express the equation of the geodesic line independently of the coordinate using the Christoffel symbols (Einstein, 1914, 1044ff.):

$$
\frac{d^{2} x_{\tau}}{d s^{2}}=\sum_{\mu \nu}\left\{\begin{array}{c}
\mu \nu \\
\tau
\end{array}\right\} \frac{d x_{\mu}}{d s} \frac{d x_{\nu}}{d s}
$$

After giving presentation of the hole argument in $\S 12$, in $\S 13$ Einstein still restricts the covariance only to linear transformations in order to ensure a unique relation between $g_{\mu \nu}$ and $T_{\mu \nu}$ (Einstein, 1914, 1066ff.).

2.2. Generally Covariant Field Equations. In the meantime the theory, that had initially received rather suspiciously by physicists, began to attract the interest of mathematicians such as Levi-Civita himself (Cattani and De Maria, 1989) and David Hilbert (Corry, 2003). Under the pressure of a hasty competition with the latter, by November of 1915 Einstein regained general covariance for its field equations, which he had abandoned "with a heavy heart" (Einstein, 1915d, 778) three years before and presented them in four communications to the Prussian Academy (Einstein, 1915c,d,a,b). In the first of three papers presented to the Berlin Academy he famously described General Theory of Relativity: as "a real triumph of the method of the general differential calculus founded by Gauss, Riemann, Christoffel, Ricci, and Levi-Civiter [sic]" (Einstein, 1915d, 778).

Einstein came first to consider again the "Ricci tensor," $R_{\mu \nu}$ as the left-side of his equations, that had to be equated to the matter tensor $T_{\mu \nu}$. The matter tensor is however divergence-free, expressing the conservation of energy, stress, and momentum, whereas the divergence of the Ricci tensor is generally non-zero. Einstein discovered that the combination $G_{\mu \nu}=R_{\mu \nu}-\frac{1}{2} R g_{\mu \nu}$ (where $R=g^{\mu \nu} R_{\mu \nu}$ ) is divergence-free. The tensor $G_{\mu \nu}$ (later labeled "Einstein tensor") has all the required properties is therefore suitable for a role of the gravitation tensor of the final field equations: $G_{\mu \nu}=-\kappa T_{\mu \nu}$ (Mehra, 1974; Pais, 1982, 20). As Einstein explained to Hilbert in November 1915:

The difficulty was not in finding the generally covariant equations from the $g_{\mu \nu}$ for this is easily achieved with the aid of the Riemann's tensor.

\footnotetext{
${ }^{3}$ Einstein discovered that generally covariant field equations it is impossible to achieve "a univocal determination of the $g_{\mu \nu}$ out of the $T_{\mu \nu}$ " (CPAE 5, Doc. 484; Einstein to P. Ehrenfest, second half of November 1913), the tensor representing the matter source. Not only general covariance allows to construct two solutions of the gravitational field equations $g_{\mu \nu}(x)$ the $g^{\prime}{ }_{\mu \nu}\left(x^{\prime}\right)$ in different coordinate systems $x$ and $x^{\prime}$ (which very first thing that one learn from absolute differential calculus), but also two solutions $g_{\mu \nu}(x)$ and $g^{\prime}{ }_{\mu \nu}(x)$ in the same coordinate system $x$; the field equations seem to attribute different field values of the field quantities for the same point (identified through unprimed coordinate system), violating the law of causality (see for instance Norton, 1987).
} 
Rather, it was hard to recognize that these equations are a generalization, and precisely, a simple and natural generalization of Newton's law. It has just been in the last few weeks that I succeeded in this (I sent you my first communication $)^{4}$ whereas 3 years ago with my friend Grossmann, I had already taken into consideration the only possible generally covariant equations, which have now been shown to be the correct ones (Einstein to Hilbert, November 18, 1915; CAPE 8a, Doc. 148, 201).

An important step toward the final breakthrough is usually considered the overcoming of what Einstein famously called a "faithful prejudice" [ein verhängnisvolles Vorurteil] (Einstein, 1915d, 782) "The key to this solution", was found, as he wrote in a letter to Sommerfeld, when Einstein (starting from November 1915) came to realize that the negative Christoffel-symbols (of the second kind) $\Gamma_{\mu \nu}^{\tau}=-\left\{\begin{array}{c}\mu v \\ \tau\end{array}\right\}$ are "to be regarded as the natural expression of the gravitational field "components", (28.11.1915; CPAE, 8a, Doc. 153, 207-208; Norton, 2003; Janssen, 2008).

A free material point moves with uniform motion in a straight line, relative to a system in which the $g_{\mu \nu}$ are constant $\left(\Gamma_{\mu \nu}^{\tau}=0\right)$. If a new co-ordinates is smoothly introduced, the $g_{\mu \nu}$ will no longer be constant, but functions of the coordinates $\left(\Gamma_{\mu \nu}^{\tau} \neq 0\right)$; the motion of the free material point will present itself in the new coordinates as curvilinear non-uniform motion. Via the principle of equivalence, we can equally interpret this motion as a motion under the influence of a gravitational field.

As Einstein summarizes in the final 1916 "polished" presentation of the theory, published in the Annalen der Physik (Einstein, 1916b), it is then natural to extend this reasoning to the case "when we are no longer able by a suitable choice of coordinates to apply the special theory of relativity to a finite region", that is when the $\Gamma_{\mu \nu}^{\tau}$ cannot be made to vanish identically, since Riemann-Christoffel tensor $R_{\mu \nu \tau}^{\rho}$ does not vanish (Norton, 1985).

Interestingly, even if Einstein does refer to the non-Euclidean geometry, in the 1916 paper the Riemann-Christoffel tensor $R_{\mu \nu \tau}^{\rho}$ does not carry most of the geometrical implications that we take for granted today; in particular Einstein does not refer to the "curvature of spacetime" (Reich, 1994, 204f.). The Riemann tensor is introduced in so far that it is the only tensor that can be constructed solely from the fundamental tensor without going beyond the second derivatives of the $g_{\mu \nu}$ (as the analogy with the Poisson's equations requires).

A "geometrical" issue emerges rather when one has to compare the values of the $g_{\mu \nu}$ predicted by the field equations (let say the Schwarzschild-solution) with the observed values. Till the end of his life Einstein insisted on assuming "provisionally" (Howard, 1990, 2005) that these can be obtained by direct measurement using small rods and atomic clocks whose length and rate are independent of the gravitational field they are embedded in (Stachel, 1989). Roughly, the $g_{\mu \nu}$ are the numbers to which we have to multiply the coordinate distances so that $d s^{2}$ has the same absolute value (up to the global choice of unit of measure) everywhere on the manifold.

2.3. Critique and Geometrical Development of Absolute Differential Calculus. As early as 1916 the Austrian physicist by Friedrich Kottler, criticized Einstein for referring to the Christoffel symbols as the components of the gravitational

\footnotetext{
${ }^{4}$ Einstein, $1915 d$
} 
field (Kottler, 1916). Christoffel-Symbols are not tensors, and they can be nonzero in a flat spacetime simply by virtue of curvilinear coordinates. Responding to Kottler, Einstein suggested that the equation $\frac{d^{2} x_{\tau}}{d s^{2}}=\Gamma_{\mu \nu}^{\tau} \frac{d x_{\mu}}{d s} \frac{d x_{\nu}}{d s}$ "as a whole is generally covariant" (Einstein, 1916a, 641), but the two terms taken separately are not. In particular the first term of the geodesic equation can be taken as representing "the Galilean inertia", and the second term with the Christoffel symbols, as "representing influence of the gravitational field upon the mass point" (Einstein, 1916a, 641). Neither of these per se has physical meaning, only their "sum" does.

In Einstein's eyes the covariance principle and the equivalence principle appear then as deeply connected: the variability of $g_{\mu \nu}$ and the non-vanishing of the $\Gamma_{\mu \nu}^{\tau}$ introduced by a coordinate transformation can be interpreted arbitrarily as an acceleration field or as a (homogeneous) gravitational field: "the requirement of general covariance of equations embraces the principle of equivalence as a quite special case" (Einstein, 1916a, 641). Einstein explicitly embraced the view that the gravitational field is a coordinate-dependent quantity (Einstein, 1918a, 699f.; see Janssen, 2011).

The most famous objection against Einstein's use of the absolute differential calculus was of course that which was raised by Erich Kretschmann in 1917 (Kretschmann, 1917). The principle of "general covariance" as complete coordinate generality in the formulation of a physical theory, has no particular physical content, and thus it has nothing to do with a principle of relativity. In fact "according to Ricci and Levi-Civita's investigations" (Kretschmann, 1917, 579) every space-time theory can be formulated in a general co-variant way, only by inserting the $g_{\mu \nu}$ ad the $\Gamma_{\mu \nu}^{\tau}$ into the equations of the theory (more on this topic Rynasiewicz, 1999; Norton, 2003). In a 1918 paper, Einstein agreed with Kretschman's claim, emphasizing nevertheless the heuristic value of general covariance when combined with a principle of simplicity (Einstein, 1918c).

A more compelling answer was provided by Hermann Weyl in the first edition of Raum-Zeit-Materie (Weyl, 1918b). Even if every theory can be rewritten in a general covariant form, in pre-general-relativistic theories such as special relativity, the metric displays the pre-asseigned Minkowski values $g_{i k}$ and the $\Gamma_{h l}^{i}$ vanish everywhere; in General Relativity one finds these values only after having solved the field equations: "This seals the doom of the Idea that a geometry may exist independently of physics in the traditional sense" (Weyl, 1918b, 174).

Weyl could make this point clear exploiting the geometrical implications of tensor calculus which were developed in those years under the stimulus of General Relativity. In 1916/1917, Levi-Civita (Levi-Civita, 1916, but see also Hessenberg, 1917/18 and Schouten, 1919) had famously recognized the geometrical meaning of the Christoffel symbols as determining the parallel displacement of vectors (Struik, 1989; Reich, 1992). A displacement preserving the direction of a vector can be expressed in general coordinates precisely by the fact that the Christoffel Symbols can be made to vanish along the path; thus roughly, referring to the displacement operation, the $\Gamma_{h l}^{i}$ turned out to be expressible without referring to $g_{i k}$.

The absolute differential calculus can then be the founded "geometrically". When a vector in a Euclidean space is parallel-transported around a loop, it will always return to its original position ( $\Gamma_{h l}^{i}$ vanish overall on the manifold). However, this property does not hold in the general case. The Riemann curvature tensor measures precisely the change in the direction (but not in magnitude as in Weyl's more general 
non-Riemannian geometry Weyl 1918s) of a vector after it is transported around a closed loop (Weyl, 1918b, §16). Along a geodesic path the vector will remain 'unchanged', so that a geodesic line can be defined in a non metrical-way as the straightest line rather than the line of extremal length (Weyl, 1918b, §17).

In the third revised edition of Raum-Zeit-Materie published in 1919, the Christoffel Symbols $\Gamma_{s r}^{i}=\Gamma_{r s}^{i}$ are considered as the "components of an affine connection" (Weyl, 1919, 101). Exactly because it is not a tensor, the affine connection provides an adequate representation of the fact required by Einstein's interpretation of the equivalence principle: there is no unique decomposition of the connection into an inertial background plus a gravitational field (Stachel, 2007). In 1920 Weyl introduced the celebrated expression "guiding field" (Führungsfeld) for the affine connection: General Relativity does make every motions relative, eliminating the structure responsible for the distinction between geodesic and non geodesic worldlines; Einstein's theory rather transformed such a constraining "guidance", in a physical force-field, in which "according to Einstein, inertia and gravitation constitute a inseparable unity" (Weyl, 1920). Motion along a geodesic path or deflection from a geodesic path, are absolutely different, but can be interpreted arbitrarily as the effect of inertia or gravitation.

In 1920, in the unpublished paper Grundgedanken und Methoden der Relativitätstheorie in ihrer Entwicklung dargestellt (CPAE 7, Doc. 31). Einstein, using a celebrated analogy with electromagnetic field which can be split differently into electric and magnetic components by different observers, came to recognize that the crucial point of General Relativity is the fact that "the gravitational field only has a relative existence" (CPAE 7, Doc. 31, p. 21; Janssen, 2005, 2008).

In the Princeton Lectures, published in the same year, Einstein emphasized again that this is well represented by the fact that "the intensity of the gravitational field" is expressed by the quantities $\Gamma_{k l}^{i}$, which "do not have a tensor character" (Einstein, 1921b) and thus are coordinate-dependent. Moreover, Einstein recognized that this could become completely clear only after "Levi-Civita rightly pointed out" that it is "the infinitesimal displacement field $\Gamma_{k l}^{i}$ that should be considered as primary" and not to the metric $g_{i k}$ (Einstein, 1921b; see Stachel, 2007).

In the early 1920s, Élie Cartan, starting from the Levi-Civita's geometrical notion of parallel displacement, considered gravity, mathematically represented by the affine connection, as the structure reconciling the different orientations of local inertial frames (Cartan, 1923, 1924b, 1925). From this point of view, according to Cartan, "relativity faces the paradoxical task of interpreting, in a non-homogeneous universe, all the results of so many experiences by observers who believe in homogeneity of the universe" (Cartan, 1924a, 81). At this point, it might be said, the connection of Ricci's calculus with Riemann's original geometrical approach is restored. With General Relativity, as Levi-Civita noticed some years later, clearly hinting to Bianchi's words, "Ricci's calculus revealed itself to be not only useful but truly indispensable" (Levi-Civita, 1925, 11, tr. 1927, VII).

\section{Relativity of Coordinates vs. Relativity of Geometry: The Young ReichenbaCh's Conversion to CONVENTIONALisM}

On the philosophical side as early as March 1917 Schlick had published the article version of his classical Raum und Zeit in der gegenwärtigen Physik (Schlick, 1917a). The work appeared in the same year in the book (Schlick, 1917b), which 
received four different editions up till 1922 (see Schlick, 1922/2006). Schlick, as is well-known, exploited the geometrical implication of General Relativity in quite different way, by casting General Relativity in a conventionalist tradition, which Schlick had allegedly found in the work of Riemann, Helmholtz and, most of all, Poincaré. Spacetime is in itself metrically "amorphous [gestaltlos]", as Poincaré has argued (Schlick, 1917a, 167); a certain space or spacetime is indistinguishable from every other that by a continuous and one to one transformation, that preserves the neighborhood relations among points or events. Hence the choice among them can only be made by an arbitrary stipulation, an implication which Schlick called "the geometrical relativity of space" (Schlick, 1917a).

Einstein, as it is well known, was very pleased by Schlick's paper and his opinion was as well very positive for the book version (Howard, 1984). However, as early as 1920 in his first monograph on relativity, Hans Reichenbach, who had attended Einstein's lectures on General Relativity in Berlin in the late 1910s, had raised a rather convincing objection against a conventionalist approach to General Relativity.

Conventionalism, Reichenbach argued, works only for spaces of constant curvature. In every of such spaces there is the a unique (up to a constant positive factor - i.e. up to the choice of a "unit of length") set of congruence relations so that each such set of congruence relations is inconsistent with any other such set. Conventionalism is then based precisely on the idea that one can make an arbitrary choice among one of such sets, that is as to which figures all observers agree on as being congruent.

In Riemannian geometry of variable curvature no unique set of congruence relations can be defined all over the space, so the very idea of a unique conventional choice among alternative congruent relations does not make sense. For this reason, Reichenbach points out, Poincaré "excludes from the beginning Riemannian geometry, because it does not permit the displacement of a body without change of form" (Reichenbach, 1920b, 104, n. 1; tr. 1965, 109, 1; translation modified). In the general case only the unit of length is globally available on a Riemannian manifold (all observers can agree per convention of using, for instance, the centimeter as unit of measure), in contrast to a non-Riemannian is Weyl's geometry that where a separate unit of length at every point of space may be defined.

According to Reichenbach, Einstein's general theory, adopting the Riemannian approach, leads rather "to an absolutely objective determination of the structure of space" (Reichenbach, 1920a, 405; tr. 2006, 29). Even if we are free to choose the coordinate system, only the properties which are independent of a particular coordinate system are physically meaningful: "Relativity does not mean the abandoning of a judgment, but the liberation of the objective sense of knowledge from its distortion through our subjective nature" (Reichenbach, 1920a, 405; tr. 2006, 29).

In these few words Reichenbach seemed to catch what General Relativity had inherited from the Riemannian tradition: Riemannian geometry is formulated in such a way that it works in arbitrary coordinates. Whereas Schlick tried to cast the contribution of Riemannian geometry to General Relativity under the light of Helmholtz's and Poincaré's philosophy of geometry as the freedom of choosing among different physical geometries; Reichenbach insisted that Riemann had showed under which condition it was possible to express the same physical spacetime by different mathematical functions depending on which coordinates were used. 
3.1. Relativity of Coordinates: Reichenbach's Early Interpretation of Riemannian Geometry and of its role in General Relativity. Reichenbach's early approach reproduces quite well Einstein's original reasoning. As Einstein wrote to Schlick, "in principle there can exist finite (matter-free) parts of the world" (Einstein to Schlick, March 21, 1917; CPAE 8a 305) that can be covered by a rectangular grid of unit rods and clocks. The four-dimensional line element is expressed as the sum of the squares of the coordinate differential $d s^{2}=\sum_{1}^{4} d x_{\nu}^{2}$. If one introduces new curvilinear coordinates by means of an arbitrary smooth substitution of the independent variables, the line element will not preserve its simple form but will change into a mixed quadratic expression: $d s^{2}=\sum_{1}^{4} g_{\mu \nu} d x_{\nu} d x_{\nu}$ :

The coefficients $g_{\mu \nu}$ occurring in [the mixed quadratic differential form] manifest themselves in the acceleration of the second coordinate system relative to the inertial system; since this acceleration directly characterizes the gravitational field of the second system, we may regard it as a measure of this gravitational field. We notice, therefore, that the transition from a gravity-free field to a gravitational field is connected with a transition to non-Euclidean coordinates, and that the metric of these coordinates is a measure of the gravitational field (Reichenbach, 1920b, 23 ; tr. 1965, 24).

Reichenbach is however careful to emphasize that "[s]uch a space is only apparently non-Euclidean; actually it does not differ structurally from Euclidean space" (Reichenbach, 1920b, 23; tr. 1965, 25). It is, on the contrary, the very same flat spacetime that "can be expressed in terms of non-Euclidean coordinates" (Reichenbach, 1920b, 23; tr. 1965, 25), where the $g_{\mu \nu}$ are not constant but functions of the coordinates.

Einstein identified the presence of the gravitational field with the non-constancy of $g_{\mu \nu}$. As Reichenbach observes, the "transition is made from the special theory to the general theory of relativity", can be considered as "a far reaching extrapolation" (Reichenbach, 1920b, 24; tr. 1965, 26; my emphasis). Einstein "inferred from this that every gravitational field, not only that which is produced by transformation, manifests itself by a deviation from Euclidean geometry" (Reichenbach, 1920b, 23; tr. 1965, 24). The presence of gravitation manifests itself in the non-constancy of the $g_{\mu \nu}$, also in the case where it is not possible "to choose the coordinates in such a way that the line element becomes Euclidean at all points simultaneously" (Reichenbach, 1920b, 27; tr. 1965, 29). This is the significance of the introduction of a quadratic form with variable coefficients:

The special position of the mixed quadratic form of the line element can also be characterized in the following way. The ten functions $g_{\mu \nu}$ determining the metric are not absolutely fixed, but depend on the choice of the coordinates. They are not independent of one another, however, and if four of them are given, the coordinates as well as the other six functions are determined. This dependence expresses the absolute character of the curvature of space. The metric functions $g_{\mu \nu}$ are not relative; that is, their choice is not arbitrary (Reichenbach, 1920b, 27; tr. 1965, 29; my emphasis).

If one covers a flat space with any "any curved oblique coordinates, then the line element will become a mixed quadratic expression. Even the ordinary polar coordinates furnish an expression differing from the pure quadratic sum for the line 
element" (Reichenbach, 1920b, 24; tr. 1965, 25). Thus, even the very simple "representation of Euclidean space by means of polar coordinates can be conceived as a projection upon a non-Euclidean space" (Reichenbach, 1920b, 24; tr. 1965, 25). In polar coordinates the $g_{\mu \nu}$ are not constant. However "the Riemannian measure of curvature of this system will be zero at every point" (Reichenbach, 1920b, 23; tr. 1965, 25). The lines of the co-ordinate grid are curved, but not the surface itself. Cartesian coordinates, where the $g_{\mu \nu}$ has constant values, can be reintroduced by simple coordinate transformation. By contrast, on a non-flat non-Euclidean space "it is impossible to preserve its simple Euclidean form" (Reichenbach, 1920b, 94; tr. 1965, 99). Cartesian coordinates simply do not exist:

the four space-time coordinates can be chosen arbitrarily, but that the ten metric functions $g_{\mu \nu}$ may not be assumed arbitrarily; they have definite values for every choice of coordinates ... If the metric were a purely subjective matter, then the Euclidean metric would have to be suitable for physics; as a consequence, all ten functions $g_{\mu \nu}$ could be selected arbitrarily. However, the theory of relativity teaches that the metric is subjective only insofar as it is dependent upon the arbitrariness of the choice of coordinates, and that independently of them it describes an objective property of the physical world (Reichenbach, 1920b, 86-87; tr. 1965, 90-91)

As Reichenbach recognizes, the mathematical apparatus of Riemannian geometry is mainly concerned with the problem of establishing when different sets of $g_{\mu \nu}$ represent different geometries, and when they are merely the consequence of the coordinate system used. As we have seen, Riemann, Christoffel and Ricci had found in the so called Riemann-Christoffel tensor an absolute criterion for distinguishing the class of different $g_{\mu \nu}$-systems that differs only by a coordinate transformation from other classes.

Hence, the freedom in the choice of the coordinate system has of course nothing to do with the freedom in the choice of geometry:

It is true that the metric contains a subjective element, and depending on the choice of the system of reference, the metric coefficients will vary; this indeterminacy [Unbestimmtheit] still holds in the gravitational field. But there exist dependency relations among the metric coefficients, and if four of them are arbitrarily given for the whole space, then the other six are determined by transformation formulas. ... That something exists manifests itself in the dependency relations between the metric coefficients; since we can discover these relations by means of measurements - and only by means of them-we can discover the real. It is the essence of the general theory of relativity that the metric is much more than a mathematical measurement of bodies; it is the form by means of which the body is described as an element in the material world. (Reichenbach, 1920b, 96-97; tr. 1965, 102; my emphasis)

The existence of such indeterminacy raised a major philosophical problem that lies in the background of Einstein's hole argument and that rapidly came to appear most often in the literature of (Pauli, 1921, 56) in Hilbert's version as the first treatment of the Cauchy problem in General Relativity. Reichenbach seems here to insist rather on the fact that the existence of such non-physical degrees of freedom does not reflect any lack of determinacy of the geometrical structure of the world. Reichenbach came then to the conclusion that the metric contains a subjective aspect 
which depends on the choice of the coordinate system and objective one, which is expressed in the dependencies among the metric coefficients.

In a way somehow similar to that of Arthur Eddington (Eddington, 1920), the still "Kantian" Reichenbach emphasizes the epistemological significance of the use of tensor calculus. The philosophical meaning of the "Riemannian analytic metric" is that it presents the mathematical technique to isolate those elements which have objective physical significances, from those which are merely artifacts of the coordinates: "invariance with respect to the transformations characterizes the objective content of reality, the structure of reason expresses itself in the arbitrariness of admissible systems" (Reichenbach, 1920b, 86; tr. 1965, 90; my emphasis).

3.2. Relativity of Geometry: Reichenbach's Last Step to Conventionalism. Having the possibility to give a first look to Reichenbach's book, Schlick immediately wrote to Einstein: "Reichenbach does not see to me to be fair [nicht gerecht zu sein] toward Poincaré's conventionalism [Konventionslehre]" (Schlick an Einstein, 9.10.1920; CPAE 10, Doc. 171). Writing to Reichenbach some weeks later, Schlick tried to the debunk Reichenbach's critique of conventionalism by arguing that Poincaré in later writings included geometries of variable curvature in his approach (Reichenbach to Schlick 26.11.1920; Schlick and Reichenbach, 1920-22). Reichenbach answered agreeing on the fact that in principle one could chose between keeping relativity and abandoning Euclidean geometry or vice-verse: "physics, however, makes the first decision ... you, and Poincaré, would say for the sake of simplicity [um der Einfachheit halber] ... But I have an instinctive disinclination [Abneigung] for this interpretation" (Reichenbach to Schlick 29.11.1920; Schlick and Reichenbach, 1920-22).

Schlick's arguments must have been very persuasive. Reichenbach very rapidly overcame his "instinctive disinclination" for conventionalism (on this point see: Parrini, 2005). Einstein's epistemological achievement becomes precisely the fact that he has shown that it would have been possible in principle for physics to make the second decision, that is to get rid of non-Euclidean geometry by preserving gravitation as a real force. A significant step in this direction can be observed already in a passage of Reichenbach's 1921 review article Der gegenwärtige Stand der Relativitätsdiskussion. Eine kritische Untersuchung. In commenting on Schlick's position Reichenbach notices:

Only with regard to the arbitrariness of the co-ordinates is space as an ideal structure; metric, however, expresses an objective property of reality. This conception does not contradict conventionalism. Schlick must not be interpreted as saying that a certain metric has been prescribed; a metric emerges only after the physical laws have been established (the $P$ of Einstein's formula). One can also change the metric, provided one changes the laws of physics correspondingly (Reichenbach, 1921, 356; tr. 1878, I, 34f.; my emphasis)

Reichenbach considered Schlick's position (see Schlick's commetary in Helmholtz, 1921) as correctly reproducing Einstein's famous claim (Einstein, 1921a) that "only the sum $G+P$ of geometry and physics is testable by experience" (Reichenbach, $1921,355 ; 1878, \mathrm{I}, 33)$.

As we have mentioned, Logical Empiricists failed to notice Einstein's reference to Poincaré (Einstein, 1921a) and Helmholtz (Einstein, 1925) should be read in the 
context of Einstein's "measuring rod objection" against Weyl's theory of electromagnetism (Weyl, 1918a; Einstein, 1918b), rather as an argument for geometrical conventionalism. However, the joint authority of Einstein and Schlick was sufficient to induce Reichenbach to conclude, already in a paper published in French in 1922 (Reichenbach, 1922, tr. 2006 ), that "[t]he solution to the problem of space is therefore found only in this conception we call conventionalism and which goes back to Helmholtz and Poincaré" (Reichenbach, 1922, 40; tr. 2006; 135).

Reichenbach's position is well-known. If the measurements with rigid rods yield a non-Euclidean geometry, one could, alternatively, maintain that the geometry of space-time was Euclidean holding that measuring instruments are actually non rigid, deformed by a non-detectable force of type $X$, which causes uniform shrinkages and expansions in all materials. According to Reichenbach, the "the real problem lies in deciding between these alternatives: either Euclidean geometry and a field $X$ or the geometry determined by experience and no field $X$ " (Reichenbach, 1922, 40; tr. 2006; 135) The empirical facts can force us to select either the Euclidean or the non-Euclidean description as the uniquely correct description.

Of course, gravitation, in Reichenbach's terminology is "a force of type $X$," (Reichenbach, 1922, 41; tr. 2006; 132); as consequence of the identity of inertial and gravitational mass, it affects all bodies in the same way. Einstein's choice "to set $X=0$ " (Reichenbach, 1922, 43; tr. 2006; 129) and abandon Euclidean geometry was the most simple choice, but not the only one possible. In principle, another more complicated alternative would have been plausible. According to Reichenbach, the very existence of this alternative represent the "characteristic of the epistemological solutions for which we are indebted to the theory of relativity" (Reichenbach, 1922, 40 ; tr. 2006; 135).

When, however, Reichenbach describes in some detail why gravitation is is force of type $X$ a different "alternative" comes to fore:

It is also in this fashion that we can obtain the remarkable identification of gravitation with certain fictitious forces that result from a change in coordinates. Imagine a gravitation-free space in which we find a system of coordinates formed by a network of congruent rigid rods. In this space the metric is Euclidean; that is to say the $g_{\mu \nu}$ possess the well-known special form $\left[d s^{2}=\sum d x_{\nu}^{2}\right]$. If we now introduce new coordinates such that the rods get progressively shorter the farther to the exterior a rod is, so that the network will be considered to be curved, then the new $g_{\mu \nu}$ take a form different from the special form. This can be conceived of in the following way: there exists a force which shrinks the rods and this force is represented by the deviation of the $g_{\mu \nu}$ from the special form; it is therefore considered to be a correction in the establishment of the $d s^{2}$. It is clear that this force ... is only a fictitious force produced by the anomaly of the rods. All of the magnitudes that, in an element of the network, are measured with a local unit undergo the same correction; this is precisely the motivation for considering this fictitious force to be interpreted as a gravitational field (Reichenbach, 1922, 39; tr. 2006; 134; my emphasis).

In the example considered by Reichenbach, one is not in front of alternative geometries; it is the very same flat geometry in different coordinate systems. A matter free region of spacetime appears as devoid of gravitation from the perspective of one coordinate system and endowed with a gravitational field when considered from 
another coordinate system: the difference is one of description. Gravitation is a "fictitious force" because it is coordinate-dependent: as Reichenbach's writes "the gravitational field and the corrections resulting from a simple change in coordinates can be brought together in a single concept" (Reichenbach, 1922, 39; tr. 2006; 134).

This of course the very core of the equivalence principle as Einstein originally presented it. As Reichenbach explains, although only in a brief footnote, "it is necessary here to draw a distinction between the gravitational potential and the gravitational field", that is between "the gravitational potentials (the $g_{\mu \nu}$ )" and "[i]ts gradient, the field" (Reichenbach, 1922, 39, n.; tr. 2006; 134, n.). The components of the metric represents the "gravitational potentials", whereas, as in other field theories, the "gradient of the potentials" is the natural candidate for representing the gravitational field.

What distinguishes the gravitational field from other fields is the fact that in a flat region of space-time non-constant potentials $g_{\mu \nu}$ can be introduced by "a simple change in coordinates". The transition to the General Theory of Relativity is realized by the assumption that such a representation of the field by the nonconstancy of the functions $g_{\mu \nu}$, is also justified in the general case in which the metric cannot be reduced to quasi-Euclidean form of the special theory of relativity by "a simple change in coordinates".

In his more technical writing Axiomatik der relativistischen Raum-Zeit-Lehre (Reichenbach, 1924), Reichenbach emphasizes that what characterizes a local inertial system is the fact that the gradient of the metric $\frac{\partial g_{\mu \nu}}{\partial x_{\sigma}}$ can be made to vanish by a coordinate transformation, whereas in a general gravitational field this is impossible, as the gradient of the metric $\frac{\partial^{2} g_{\mu \nu}}{\partial x_{\sigma} \partial x_{\rho}}$ does not vanish. However Reichenbach's philosophical conclusion is stunningly different (cf. Reichenbach, 1924, 106; tr. 1965, 133).

Focusing on what he now calls "metrical forces" (Reichenbach, 1924, 68; tr. 1965, 87) (because they depend on the choice of the metric), Reichenbach is convinced that "space and time in the General Theory of Relativity mean the same as in the special theory although without any metric" (Reichenbach, 1924, 155; tr. 1965, 195); the "topological properties turn out to be more constant that the metrical one", so that Reichenbach famously argues that "the transition from the special theory to the general one represents merely a renunciation of metrical characteristics, while the fundamental topological character of space and time remains the same." (Reichenbach, 1924, 155; tr. 1965, 195)

In the immediately subsequent years, Logical Empiricists came rapidly to agree on the fact that such an opposition between the topological and the metrical properties of spacetime is the relevant innovation introduced by General Relativity. Carnap, in his first post-doctoral writings (Carnap, 1923, 1925), could easily translate into an empiricist framework his early "Kantian" conventionalism (Carnap, 1922). Only topological space reproduces what is present in experience uni-vocally. By contrast, all post-topological structure depends upon a stipulation.

It is undeniable that the idea was philosophically appealing. In every physical theory we can distinguish on the one hand a factual element determined by experience on which a class of empirically equivalent theories agree and on the other a conventional element determined pragmatically as the "simplest" theory in the class. "Simplicity" has of course no intrinsic connection with "truth". In the standard Logical Empiricist view, the development of science appeared then to be 
marked by the progressive discovery that there "are certain elements of knowledge ... which are not governed by the idea of truth, but which are due to volitional resolutions" (Reichenbach, 1938, 9; my emphasis).

\section{Reichenbach's Mature Conventionalism and the "Analytic Treatment of Riemannian Spaces"}

In 1926, Reichenbach had already finished his semi-popular Philosophie der Raum-Zeit-Lehre (1926, but published only as Reichenbach, 1928, tr. Reichenbach, 1958), which furnishes a very elegant and effective presentation of this doctrine and of the noble tradition from which it emerges:

This conception of the problem of geometry is essentially the result of the work of Riemann, Helmholtz, and Poincaré and is known as conventionalism. While Riemann prepared the way for an application of geometry to physical reality by his mathematical formulation the concept of space, Helmholtz laid the philosophical foundations. In particular, he recognized the connection of the problem of geometry with that of rigid bodies ... It is Einstein's achievement to have applied the theory of the relativity of geometry to physics. The surprising result was the fact that the world is non-Euclidean, as the theorists of relativity are wont to say; in our language this means: if $F=0$, the geometry $G$ becomes non-Euclidean. This outcome had not been anticipated, and Helmholtz and Poincaré still believed that the geometry obtained could not be proved to be different from Euclidean geometry. Only Einstein's theory of gravitation predicted the non-Euclidean result which was confirmed by astronomical observations (Reichenbach, 1928, 48; tr. 1958, $35)$.

According to what Reichenbach calls theorem $\theta$, a non-Euclidean geometry $G$ is equivalent to an Euclidean geometry $G^{\prime}$ with a field of "universal forces" $F$. Only the combination $G+F$ is testable by experience, after a conventional choice has been made. Einstein chose the most simple convention by setting $F=0$.

The action of such a force is not completely arbitrary. In order to avoid causal anomalies (Reichenbach, 1928, §12) the original geometry $G$ must be mapped into the new one $G^{\prime}$ uniquely and continuously. The very lesson that we can are draw from Einstein's theory is that we are free to chose among topologically equivalent, but metrically different spaces, that can be smoothly deformed into one-another, by some "force" $F$ that affect all bodes. The "metrical relations are distorted" (Reichenbach, 1928, 48; tr. 1958, 35), whereas the "topological" structure remained untouched. According to Reichenbach, the world as it is in-itself does not have a unique metric; it does, however, have a unique topology, and this is defined in terms of the causal relations.

Reichenbach believes that Riemann had set the mathematical framework for this philosophical conclusion. Riemann's "mathematical achievement" is "of greatest significance for the epistemological problem of space" (Reichenbach, 1928, 244; tr. 1958, 279f.). "The mathematical treatment" divides the description of a space "into a topological and a metrical part": "the function of numbering" is assigned" to the coordinate system; whereas "the metrical functions of measuring lengths" that are assigned "to the metrical coefficients $g_{\mu \nu}$ " (Reichenbach, 1928, 244; tr. $1958,280)$ on the other. In Riemannian geometry the "metrical function of the $g_{\mu \nu}$ plays a subordinate role. It cannot change the topological foundation determined by 
the coordinate system. It merely adds to it a metrical superstructure [Überbau]" (Reichenbach, 1928, 244; tr. 1958, 280).

4.1. Reichenbach's Technical Presentation of Riemannian Geometry and its Incompatibility with his own Conventionalism. In Reichenbach's view Riemann deprived the coordinate system of all but topological properties. This interpretation seems however to be based on a fundamental misunderstanding. Indeed, in Riemannian geometry coordinates have per se no metrical significance; they are only a set of markers that serve to distinguish the points. Yet such a significance is regained by the introduction of a quadratic differential form with variable coefficients $g_{\mu \nu}$ as function of the coordinates. Instead of separating the metric and topological significance of the coordinate system, Riemannian geometry shows that there is no other source of information about the coordinates apart from the $g_{\mu \nu}$.

One is free to introduce any coordination of the physical space that is produced by an arbitrary, if only, smooth transformation from the original. However, this transformation is accompanied by a suitable change of the $g_{\mu \nu}$, so that that the $d s^{2}$ are unchanged; all measured relations can be "recovered" in the new coordinate system by using the new $g_{\mu \nu}^{\prime}$ to get real distances from coordinate distances. "The numbers $g_{\mu \nu}$ indicate how, at a given place, the length of the line element is to be calculated from the coordinate differentials" (Reichenbach, 1928, 243; tr. 1958, 279).

As Reichenbach has explained in his 1920 monograph, the coordinate system is arbitrary, however the dependency of the $g_{\mu \nu}$ from the coordinate system has an objective meaning. This still emerges clearly from Reichenbach's own presentation of the "Analytic Treatment of Riemannian Spaces" in his 1926/1928 book:

This fact is expressed analytically by a property of the $g_{\mu \nu}$, as we shall see when we investigate transformations to other coordinate systems. Let us imagine that a second coordinate system has been introduced and that the position of the new family of lines is given as a function of the old coordinates, ... We now add the restriction that the transition to the new coordinates must not change any of the metrical relations; this transition, therefore, leads only to a different form of description. We must then specify a new system $g_{\mu \nu}^{\prime}$ of metrical coefficients relative to the new coordinates $x_{\mu}^{\prime}$ such that the old relations of congruence are preserved. If two line segments are equally long when measured by the old $g_{\mu \nu}$ in the old coordinate system, they must still be equally long when measured by the new $g_{\mu \nu}^{\prime}$ in the new coordinate system. This requirement leads to the condition that

$$
d s^{2}=g_{\mu \nu} d x_{\mu} d x_{\nu}=g_{\sigma \tau}^{\prime} d x_{\sigma}^{\prime} d x_{\tau}^{\prime}
$$

We can therefore say that $d s^{2}$ is an invariant of the transformation, and we can show from $(8)$ how the new $g_{\mu \nu}$ are to be calculated from the old ones. (Reichenbach, 1928, 281; tr. 1958, 245; my emphasis)

As we have seen, a classical example is of course the passage from using Cartesian (rectangular) coordinates to polar coordinates. The points are relabeled, that is labeled with different numbers (for instance, in a surface the point whose Cartesian coordinates are $(1,1)$ has polar coordinates $\sqrt{2}, \pi / 4)$. However, regardless of the coordinate system used, the distances between any two pairs of point are assigned 
the same value. The same flat Euclidean metric is expressed in a different coordinate system.

Geometrical conventionalism, violated exactly the condition, which in Riemann's approach should be considered as fundamental. This emerges clearly from Reichenbach's attempt to cast the loose language of universal forces in a more formal framework: if the results of measurement yield a metric $g_{\mu \nu}$ which is not-Euclidean, then one can infer that the geometry is actually given by the normal matrix $\overline{g_{\mu \nu}}$, if one stipulates that our measuring rods were under the influence of a universal force $F$.

Generally the force $F$ is a tensor. If the $g_{\mu \nu}^{\prime}$ are the metrical coefficients of the geometry $G^{\prime}$ and $g_{\mu \nu}$ those of $G$, the potentials $F_{\mu \nu}$ of the force $F$ are given by $g_{\mu \nu}^{\prime}+F_{\mu \nu}=g_{\mu \nu}$. The measuring rods furnish directly the $g_{\mu \nu}^{\prime}$; the $F_{\mu \nu}$ are the "correction factors" by which the $g_{\mu \nu}^{\prime}$, are corrected so that $g_{\mu \nu}$ results. The universal force $F$ influencing the measuring rod is usually dependent on the orientation of the measuring rod (Reichenbach, 1928, 44, n.; tr. 1958, 33).

Let the $g_{\mu \nu}^{\prime}$ represent some non-flat geometry; then the introduction of the arbitrary potentials $F_{\mu \nu}$ of a universal force field leads us to conclude that the metric of the space can be reduced to an Euclidean flat one $g_{\mu \nu}: d s^{2}=g_{\mu \nu} d x_{\mu} d x_{\nu}=\left(g_{\mu \nu}^{\prime}+\right.$ $\left.F_{\mu \nu}\right) d x_{\mu}^{\prime} d x_{\nu}^{\prime}$ (Norton, 1994). The equation deals precisely with a $g_{\mu \nu}$ system which cannot be transformed into $g_{\mu \nu}^{\prime}$ by a coordinate transformation if the line element has to be preserved. In fact, if this equation is valid, than trivially the quadratic form is not an invariant $d s^{2}=g_{\mu \nu} d x_{\mu} d x_{\nu} \neq g_{\mu \nu}^{\prime} d x_{\mu}^{\prime} d x_{\nu}^{\prime}$. If two tracts are congruent when measured by the old $g_{\mu \nu}$ in the old coordinate system, then they are not equally long when measured by the new $g_{\mu \nu}^{\prime}$ in the new coordinate system. Thus the two geometries can be transformed into one another only if one renounces the invariant character of $d s^{2}$; in other words,one deprives space of its metrical properties i.e. the structure which tells how much space or time lies between pointevents.

4.2. Reichenbach on Riemann's Äquivalenzproblem. There is a sort of conflict between Reichenbach's philosophical intentions and Reichenbach's own popularization of the mathematical apparatus of Riemannian geometry. Philosophically Reichenbach attempted to interpret Riemann's approach from the perspective of Helmholtz's and Poincaré's problem of finding a criterion for making a choice among different metrical geometries which only agree on the "topological order of all spacepoints determined through the coordinate system" (Reichenbach, 1929b, 683). Riemann's main concern, however, was on the contrary thereof, namely that of finding a criterion to discern the properties of the same metrical geometry that depend on the choice of a coordinate system from those that are coordinate independent. This can be understood only if one considers Riemann's geometrical insight from the perspective of the non-geometrical development put forward by Christoffel and Ricci.

From this vantage point, as again Reichenbach explains very clearly in the less philosophical parts of the book, it appears that Riemann wanted to investigate different classes of quadratic differential forms. Each class, insofar that the line element may be transformed into one another by simple coordinate transformation, represents the same geometry. On the contrary $g_{\mu \nu}$-systems which cannot be transformed into one-another by any change of the coordinates represents a different geometries: 
We may restate our results as follows: any given system $g_{\mu \nu}$ can be transformed into another system $g_{\sigma \tau}$, by means of [a smooth change of variables]. Transformations of this kind, starting with a definite set $g_{\mu \nu}$ do not give us all conceivable systems, however, but merely a limited class. The systems of this class are geometrically equivalent to the initial $g_{\mu \nu}$, and the class as a whole characterizes a definite geometry. Other classes similarly constructed, would characterize another geometry. A special class is the class which contains the normal system; it is the class of Euclidean geometry (Reichenbach, 1928, 282f.; tr. 1958, 246).

The very purpose of Riemann's investigation was to decide when two $g_{\mu \nu}$-systems differ only by a coordinate transformation and are therefore geometrically equivalent. Two metrics are equivalent, if and only if there is a coordinate transformation that transforms $g_{\mu \nu}$ into $g_{\mu \nu}^{\prime}$ so that $d s^{2}=d s^{\prime 2}$. This is of course precisely the Äquivalenzproblem. As we have seen, it is mainly the merit of Christoffel and Ricci, having developed the mathematical technique for dealing with this problem. Reichenbach describes it once again very accurately:

The question now arises whether there exists a special characteristic of the class of Euclidean Geometry. Mathematicians have shown that it is possible to formulate such a criterion. For this purpose one has to form a certain mathematical combination of the $g_{\mu \nu}$ and $g_{\mu \nu}, \frac{\partial g_{\mu \nu}}{\partial x_{\sigma}}, \frac{\partial^{2} g_{\mu \nu}}{\partial x_{\tau} \partial x_{\sigma}}$ which is called $R_{\mu \nu \sigma \tau} \ldots R_{\mu \nu \sigma \tau}$ and is therefore a tensor of rank 4. We can recognize directly ... a very important property of all tensors, namely, that if all components of a tensor are zero in one coordinate system, they will all be zero in every other coordinate system. ... Since it can be shown that $R_{\mu \nu \sigma \tau}$, vanishes for the normal system, it follows that every system of the Euclidean class is characterized by the condition

$$
R_{\mu \nu \sigma \tau}=0
$$

$R_{\mu \nu \sigma \tau}$ is called the Riemannian curvature tensor. It is a measure of curvature. (Reichenbach, 1928, 283; tr. 1958, 246)

As we have seen, $R_{\mu \nu \sigma \tau}$ entails the first and second derivatives of the $g_{\mu \nu}$. Since it is a tensor, if it vanishes in one coordinate system, it vanishes in all coordinate systems. All inter-transformable $g_{\mu \nu}$-systems represent the same Euclidean geometry, since in all these cases, even if the $g_{\mu \nu}$ can be variable, the Riemann tensor vanishes. On the contrary the Christoffel symbols $\Gamma_{\mu \nu}^{\tau}$, which entail the first derivatives of the $g_{\mu \nu}$, are not tensors. If they vanish in one coordinate system, they do not vanish in another. Hence we cannot expect that the $g_{\mu \nu}$, or the $\Gamma_{\mu \nu}^{\tau}$, should give something of absolute significance. We have to differentiate the $g_{\mu \nu}$ twice before we arrive at something that has a significance independent of any special coordinate system.

\section{Relativity of Geometry vs. Relativity of Gravitation}

Reichenbach is philosophically convinced the that "metrical properties of the space-time continuum are destroyed by gravitational fields" (Reichenbach, 1928, 269 ; tr. 1958, 308); since an alternative to Einstein-stipulation would have been in principle possible, space-time has no definite metrical structure. However, once again, following Reichenbach's semi-technical presentation of General Relativity, rather than his philosophical interpretation, one finds a more humble truth: the gravitational fields, merely "destroy the orthogonal form of the line element" (Reichenbach, 1928, 289; tr. 1958, 253). 
Let consider again an inertial frame $K$ without a gravitational field, that is where "the $g_{\sigma \tau}^{\prime}$ will satisfy the normal matrix. If we now describe the same local world region from an accelerated system $K$, the $g_{\mu \nu}$ of this system can no longer satisfy the normal matrix"; hence "the $g_{\mu \nu}$ will characterize the acceleration of $K$ " (Reichenbach, 1928, 288; tr. 1958, 253); but, because of the equivalence principle "[i]f they characterize the state of acceleration of $K$," (Reichenbach, 1928, 289; tr. $1958,253)$, "they must also characterize the gravitational field which exist for $K$ ". Of course the geometry of space has not changed in passing from $g_{\sigma \tau}^{\prime}$ to $g_{\mu \nu}$ rather it is the same flat Minkowski geometry, represented in different coordinate systems (Reichenbach, 1928, 289; tr. 1958, 253).

5.1. The Equivalence Principle and the Christoffel-Symbols. As Reichenbach already briefly explained in a footnote in his 1922 article, Einstein identified the gravitational field with the gradient of the potentials $g_{\mu \nu}$, that is with the nonvanishing of the Christoffel symbols $\Gamma_{\mu \nu}^{\tau}$. As in every field theory also in gravitational theory one distinguishes the "concepts of potential and gradient"; in electricity theory, the force field is the gradient of the electric potentials; analogously in General Relativity "the gravitational force will ... be characterized by the potential gradient [Potentialgefälle] which can be calculated for every point from the potential field" (Reichenbach, 1928, 268; tr. 1958, 233).

According to Reichenbach "[t]his representation explains why the gravitational field can be transformed away" (Reichenbach, 1928, 271; tr. 1958, 236). As we have seen, in Einstein's original approach, within a small enough region of spacetime, one can introduce an "artificial gravitational" field by a simple change of coordinate system, in which the $g_{\mu \nu}$ are not constant, but become functions of the coordinates. For the very same reason one can make this gravitational field disappear through a coordinate transformation: we can set "the metrical field in such a manner that the components, the gravitational potentials $\left[g_{\mu \nu}\right]$ become constants (this is always possible at least for local regions); then there exists no gravitational gradient. The disappearance of the gradient is then called 'the disappearance of the gravitational field." (Reichenbach, 1928, 271; tr. 1958, 236; second emphasis mine).

Reichenbach suggested then to distinguish among the tensor as $g_{\mu \nu}$ "as a whole or the metrical field", "the particular sets of tensor components", in a certain coordinate system and "finally the particular set of gradient coefficients of the tensor components" (Reichenbach, 1928, 271; tr. 1958, 236):

In the mathematical representation, the metrical field is given by the tensor $g_{\mu \nu}$ the gravitational potential field by the particular set of components $g_{\mu \nu}$, and the gravitational gradient field through the RiemannChristoffel symbols $\Gamma_{\mu \nu}^{\tau}$, which are obtained from the $\frac{\partial g_{\mu \nu}}{\partial x_{\tau}}$. The $\Gamma_{\mu \nu}^{\tau}$, do not form a real tensor, only a linear tensor, and can therefore all at once be transformed to zero by nonlinear transformations. A fourth concept has occasionally been introduced. We set $\overline{g_{\mu \nu}}+\gamma_{\mu \nu}$, where $\overline{g_{\mu \nu}}$ are the normal orthogonal values of the $g_{\mu \nu}$, and we refer to the $\overline{g_{\mu \nu}}$ as the inertial field and only to the $\gamma_{\mu \nu}$ as the gravitational potential field. The $\Gamma_{\mu \nu}^{\tau}$ may then be considered as the derivatives of the $\gamma_{\mu \nu}$, since the $\overline{g_{\mu \nu}}$ as constants do not contribute to the gradient field. This resolution into inertial and gravitational field is an adaptation to the terminology of Newtonian mechanics, however, and is therefore hardly appropriate. (Reichenbach, 1928, 272; tr. 1958, 237) 
This passage is curiously written in smaller characters; Reichenbach's intention is simply to introduce some mathematical technicalities. This is revealing of Reichenbach's philosophical attitude. From today's point of view, the passage just quoted in fact seems to describe the very conceptual core of Einstein's path to the Theory of General Relativity. The conceptual difference between gravitational "fields" and the other "fields" is that the latter, according to Einstein, should be represented by a non-tensorial, coordinate-dependent quantity, the Christoffel symbols $\Gamma_{\mu \nu}^{\tau}$. Locally, the phenomena of gravity and acceleration were, in Einstein's view, two ways of looking at the same space-time in terms of different coordinate systems.

Reichenbach is so aware of the importance of this point, that he even addresses the problems which arise following Einstein's original approach: "If we change to three-dimensional polar coordinates, for example, while the time coordinate remains unchanged, the $g_{\mu \nu}$ will assume a non-standard from. For these coordinates there must therefore exist a gravitational field", since in polar coordinates "the partial derivatives $\left[\frac{\partial g_{\mu \nu}}{\partial x_{\tau}}\right]$ do not vanish throughout" (Reichenbach, 1928, 290, n.; tr. 1958, 253). This is however rather counter-intuitive since no change in the state of motion is provided by a mere spatial transformation, which leaves the $g_{44}$ unchanged (see for instance Reichenbächer, 1923). This "says more than was originally expressed by the principle of equivalence." (Reichenbach, 1928, 289; tr. 1958, 253.)

It would therefore be advantageous to express the gravitational field in a coordinate invariant form (on this point see Eddington, 1923, 39f.): "all $g_{\mu \nu}$-systems derived from a $g_{\sigma \tau}^{\prime}$-system by means of coordinate transformations are merely different resolutions of the same tensor into different sets of components. This tensor, the metrical field, is therefore independent of specific coordinate systems" (Reichenbach, 1928, 289; tr. 1958, 253). A physical magnitude expressed by a tensor has definite components once a basis is given in a chosen coordinate system, but abstractly considered, it stands for its components in all coordinate systems. All transformable systems should represent the same gravitational field, whose presence should be better expressed by the non-vanishing of Riemann-Christoffel tensor $R_{\mu \nu \sigma \tau}$. However, this would mean "to accept the consequence that transformations of the state of motion will not change the gravitational field either, since they too leave the metrical field invariant" (Reichenbach, 1928, 291; tr. 1958, 254). In this way the "principle of equivalence" (that is the possibility of interpreting locally an acceleration field as gravitational field) would become useless.

5.2. Co-variant and Invariant. As Reichenbach notices in the popular book Von Kopernikus bis Einstein. Der Wandel unseres Weltbildes (Reichenbach, 1927) all of this can be understood precisely because Einstein "had to introduce in physics a new mathematical method, the so-called tensor calculus". "The essence of the new method of calculation resides in two basic concepts, the invariant and the covariant" (Reichenbach, 1927, 105). In particular Reichenbach suggests to interpret the gravitational field as a covariant magnitude, which depends on the coordinate system, and the metric field as an invariant magnitude:

This consideration leads to a distinction which we have touched upon several times before and which expresses a basic idea of modern science. The system of the tensor components is covariant, i.e. it has a different numerical composition for each coordinate system. Yet we express in this fashion a state that is independent of the coordinate system, i.e., an invariant state. The tensor as a whole is an invariant magnitude. We can 
recognize this property from its representation by means of components, since the components can be calculated for every coordinate system, if they are known for one. It is unfortunate that the physical terminology does not reflect this well-defined mathematical distinction. By "gravitational field" we understand the system of components of the tensor in each case; this makes the gravitational field a covariant magnitude. No particular term has been accepted for the invariant tensor field as a whole. It might best be called the metrical field, in accordance with some ideas which we shall discuss later; in fact, this term has occasionally been used with this meaning. In this terminology the gravitational field is the particular system of components into which the metrical field has been resolved (Reichenbach, 1928, 271; tr. 1958, 236).

Reichenbach's terminology is surely non-standard. As we have seen, Reichenbach himself notices that the gravitational field is represented by the Christoffel symbols, that is, by a non-tensorial, i.e. non-covariant quantity (cf. above p. 12). However, the point Reichenbach wants to make is clear enough: The values of the components $g_{\mu \nu}$ depend on a particular coordinate system, they are covariant quantities. However, regardless of the coordinate system used, the lengths of lines are assigned the same value, that is the length between a pair of spacetime points is an invariant quantity.

According to Reichenbach " $\mathrm{t}]$ he coordinate systems themselves are not equivalent", in the sense that in every system a different set of tensor components $g_{\mu \nu}$ is defined; however "every coordinate system with its corresponding gravitational field is equivalent to any other coordinate system together with its corresponding gravitational field" (Reichenbach, 1928, 272; tr. 1958, 237 ). The ensemble of all inter-transformable $g_{\mu \nu}$-systems represent the same metric field and thus the same geometry of space-time: "Each of these covariant descriptions is an admissible representation of the invariant state of the world" (Reichenbach, 1928, 272; tr. 1958, 237 ). Similarly an electromagnetic field as whole transforms as a tensor, but not the electric and the magnetic field separately: an electric field for one observer could be a superposition of an electric and a magnetic field for the other.

Surprisingly from Reichenbach semi-technical presentation of the General Theory of Relativity one learns that "the gravitational field is deprived of its absolute character and recognized as a covariant magnitude" (Reichenbach, 1928, 248; tr. 1958,214 ), that is (in Reichenbach's parlance) a coordinate-dependent magnitude, represented by $\Gamma_{\mu \nu}^{\tau}$. In a flat Minkowski spacetime, an "artificial" gravitational field can be introduced by a mere coordinate transformation. On the other hand, the geometry of space-time has an absolute character in the sense that it is coordinate independent, it does not change if one represents it in different coordinate systems; for instance, Minkowski space-time is flat, which means that the Riemann tensor $R_{\mu \nu \sigma \tau}$ vanishes everywhere regardless of the coordinate system used.

Following the more expository parts of Reichenbach's book one discovers that there is indeed a conventional element in the General Theory of Relativity; however this is not the geometry, but rather the gravitational field. The same space-time geometry, which in a coordinate system only has an inertial component, whereas in another coordinate system it has both an inertial and a gravitational. Since the Christoffel-symbols are not tensors, if they have zero components, that is only inertial components, in one coordinate-system, there exists a coordinate system in which the components are non-zero and a gravitational field appears 
The consequence is that the distinction between inertia and gravitation is a mathematical artifact which depends on the choice of the coordinates and therefore does not reflect a real physical difference: only the sum of the two pieces, represents something physical. Following this line of thought, which has remained somehow hidden behind the curtains of Reichenbach's "official" philosophy, one discovers, that the core of Einstein's original approach should not be found in the discovery of the relativity of geometry, but rather in the discovery of relativity of the gravitational field (Janssen, 2005).

5.3. "Displacement Space" vs. "Metrical Space". From this point of view Reichenbach's idea that the gravitational force has to be set equal to zero, is at least ambiguous (Torretti, 1983, 237). The best way to understand the fact gravity is a "universal force" is precisely to recognize that the gravitational field $\Gamma_{\mu \nu}^{\tau}\left(x_{\nu}\right)$ is not a tensor field on spacetime, whereas the potential field $g_{\mu \nu}\left(x_{\nu}\right)$ is. This distinction of course gets lost using the conceptual resources of the Hemholtz/Poincarés debate on the foundation of geometry; it is only "accessible to the mathematical treatment by means of Riemannian geometry" (Reichenbach, 1928, 290; tr. 1958, 253). In particular, as Reichenbach knows very well of course, Christoffel and Ricci furnished Einstein the mathematical tools to express this distinctive feature of the gravitational field.

The conventionalist approach to understand Einstein's theory in terms of relativity of geometry, of a renunciation of metrical characteristic, appears then curiously at odds with Reichenbach's own exposition. On the contrary, one has to go, so to say, beyond the metrical aspect in order to capture Einstein's conception of what has been called the relativity of the gravitational field.

The nature of Einstein's original formulation of the equivalence principle emerges in fact much more clearly by following Weyl's recognition (see above p. 12) of "the independence of the displacement operation [Verschiebungsoperation] is given by the $\Gamma_{\mu \nu}^{\tau}$ from the metric that is given by the $g_{\mu \nu}$ " (Reichenbach, 1929b, 683). Following Lev-Civita, Weyl "discovered" the independence of what Reichenbach's calls "displacement space" (Verschiebungsraum, what Weyl called "affine connection"), based on the comparison of the direction of two vectors $\left(d A^{\tau}=-\Gamma_{\mu \nu}^{\tau} A^{\mu} d x^{\nu}\right)$ in respect to the "metric space", based on length comparison $\left(l^{2}=g_{\mu \nu} A^{\mu} A^{\nu}\right)$ (Reichenbach, 1929b, 684).

From this point view it becomes particularly clear that the displacement space, the "guiding filed" that tells particles how to go is not arbitrary. What is arbitrary is the splitting of the displacement space into gravitational and inertial parts. The same path of particles can be interpreted as a consequence of the effect of inertia or of gravitation, depending on the coordinate system chosen. The difference between inertial and gravitation is a mere mathematical difference, that has no correspondence in physical realty.

However, as the last part of Reichenbach's book (which was not translated into English) was dedicated to a very careful discussion of Weyl's theory of electromagnetism, Reichenbach seems to consider this step from the metrical to the displacement field as philosophically irrelevant. The quantities $\Gamma_{\mu \nu}^{\tau}$ cannot be measured directly by rods and clocks, but must be obtained from the directly measured quantities $g_{\mu \nu}$ by calculation: they are a "product of fantasy, mere illustration" (Reichenbach, 1928, 352; on this point see Coffa, 1979). 
Contrary to Reichenbach's opinion, however, it cannot be denied that here one is confronted with one of the most relevant conceptual issues raised by General Relativity. It is well-known that modern relativists usually prefer to identify the presence of a gravitational field with the non-vanishing of the Riemann-Christoffel tensor, that is with the "curvature" (Synge, 1970) because of its independence from the coordinate system (on this topic see Lehmkuhl, 2008). The equivalence principle is then reduced to a sort of "midwife" (Synge, 1960) at the birth of General Relativity (see also Ohanian, 1977).

On the contrary, Einstein till the end of his life famously insisted that "what characterizes the existence of a gravitational field ... is the non-vanishing of the $\Gamma_{i k}^{l}$, not the non-vanishing of the $R_{i k l m}$ " (Einstein to Max von Laue, September 1950; translated in Stachel, 1986, 1858). In one of his last letters to Michele Besso in 1954, Einstein summarizes:

(This was still not so clear at the time of the setting up of the G.R., but was subsequently recognized principally through Levi- Civita). In the setting up of the theory, I chose the symmetric tensor $g_{i k}$ as the starting concept. It provided the possibility of defining the "displacement field" $\Gamma_{i k}^{l}$, which determines to every vector in the infintesimal point $P$ another vector in every infinitesimally near point $P^{\prime}\left(\delta A^{\nu}=-\Gamma_{\sigma \tau}^{\nu} A^{\sigma} d x_{\tau}\right)$

This concept of the displacement field is in-itself independent from the existence of a metric field $g_{i k}$; that it was at first introduced only in connection to the metric field, is only the consequence of the fact that Riemann moved from Gauss's theory of surfaces ... if the displacement field is introduced as a fundamental quantity, the curvature tensor is determined thorough the invariant act of displacing a vector along the boundary of infinitesimal surface-element. To the $\Gamma$-field pertains then the curvature tensors $R_{k l m}^{i}$ and $R_{k l}$ (Einstein, 1972, 525-527; partially translated in Norton 2002)

The vanishing of Christoffel symbols does not mean that there is no displacement field/gravitational (Giulini, 2001, §9). A gravitational field can always be introduced, even in a flat Minkowski space-time, by a simple change in coordinates. Thus the flat metric of spacetime can be regarded as a special case of a gravitational field, rather than the absence of a gravitational field (see also Einstein to Becquerol, August 16, 1951, cited by Norton, 1985). This seems to be in harmony with Einstein's famous claim that there is no "space without a field". If we imagine the gravitational field to be removed, there remains "absolutely nothing, and also no "topological space"' (Einstein, 1952, 155).

\section{Conclusion. Over-Determination versus Under-Determination}

Reichenbach's idea that "the topology of space is to be regarded as a more fundamental determination than the metric" (Reichenbach, 1929a) seems to derive from the observation, that the metric relations, the lengths of worldlines do not remain invariant under a deformation of spacetime (induced for instance by a universal force) that preserve smoothness of the coordinate system and the uniqueness of the labeling of the points, that is, that which deprives the coordinate system of all but is topological properties.

However distances $d o$ remain invariant under a transformation which represents a mere recoordinization, that is, under the condition that the coefficients of the quadratic form also change. Of course this is exactly the relevant point. The 
geometry of a given space-time is characterized by the invariant interval between any two world points; once a unit of measure has be chosen, the numerical value of this interval remain unchanged under coordinate transformations.

What is relevant in GeneralRelativity, as Reichenbach had shown in his 1920, is precisely the "relativity of coordinates" and not the "relativity of geometry" as in Rechenbach's mature philosophy: "The relativity of geometry is a consequence of the fact that different geometries can be represented by one another with a oneto-one correspondence" (Reichenbach, 1929a). The relativity of coordinates is that the same geometry can be represented in different coordinate systems.

As we have tried to show, the origin of Reichenbach's "conversion" can be regarded as the result of a "collision" of mathematical traditions. Instead of following the line Riemann-Christoffel-Ricci-Einstein, as he seemed to do in his first monograph, Reichenbach, probably under the influence of Schlick, tried to create the tradition Riemann-Helmholtz-Poincaré-Einstein:

The solution to the problem of space described here is to be attributed principally to the work of Riemann, Helmholtz, Poincaré, and Einstein, Helmholtz. The first to acknowledge the significance of Riemann's idea for physics, indisputably deserves the major credit for the recognition of the definitional character of congruence in physical space. Poincaré coined the term conventionalism, which refers to the definitional character of the congruence of line segments and designated the definition in question as a convention. At the time he introduced this idea, Poincaré still believed that the convention of the rigid body led to Euclidean geometry, not knowing that Einstein was soon to take up the idea of conventionalism in all seriousness and apply non-Euclidean geometry to physics, final clarification came about with the philosophical discussion of Einstein's general theory of relativity (Reichenbach, 1929a, 60; tr. Reichenbach, 1978, 179)

As recent literature has abundantly shown, General Relativity cannot be the heir of this philosophical/mathematical tradition simply because such does not exist. Poincaré excluded explicitly the Riemanniann geometries of variable curvature from his conventionalism, precisely because they were at odds with the Helmholtzian approach based on the existence of rigid bodies. As we have tried to point out, Riemann's geometrical approach to geometry remained actually dormant and developed in a rather non-geometrical tradition, whose main protagonists can be considered to be Christoffel and Ricci. The geometrical implication of the calculus that developed mainly after the emergence of General Relativity by Levi-Civita and others.

A much more plausible line of development of Riemann's work in the 19th century is for instance that which was suggested by Cartan in a non-technical paper of 1931:

Euclidean geometry itself also uses analytic methods lying on the use of coordinates, but these coordinates (Cartesian, rectangular, polar, etc.) have a precise, quantitative geometric significance, which is why they can be introduced only after the geometry is founded by its own methods. In Riemannian geometry, on the contrary, coordinates, introduced from the beginning, serve simply to relate empirically the different points of space, and geometry has precisely the object of extricating the geometric properties that are independent of this arbitrary choice of coordinates. ... The necessity of using systems of arbitrary coordinates exerted a profound influence on the later development of mathematics and physics. 
It led to the admirable creation of an absolute differential calculus by Ricci and Levi-Civita, which was the instrument that helped to elaborate general relativity (Cartan, 1931, 397f., tr. Pesic, 2007, 182).

We are dealing with two mathematical traditions which seem to be interested in different philosophical problems. Helmholtz and Poincaré tried to determine under which condition it is possible to make a choice among alternative physical geometries, which shares some weaker mathematical structure. Riemann raised rather the problem of distinguishing the intrinsic features of a given physical geometry, from the apparent differences introduced by the choice of a particular coordinate system.

There is a broader philosophical lesson that we can draw from the historical reconstruction we have outlined. Considered under the light of he 19th century philosophy of geometry the Logical Empiricists could interpret General Relativity as a case of mathematical undertermination: the relevant geometric structure that General Relativity has at its disposal (what the Logical Empiricist call the "topological" structure) is not "rich enough" to allow a choice among different possible physical geometries. Considered under the historical perspective we have suggested, the requirement of general covariance, if it is not be considered trivial, seems to raise rather a problem of overdetermination; that is, the physical system is described by a surplus of mathematical structure (Norton, 2003; more in general on this topic Redhead, 2001).

The Logical Empiricists' strategy was to introduce some redundant physical elements (the universal forces) in order to extract the mathematically relevant content of the theory. From a contemporary prospective, by the contrary, General Relativity seems rather to introduce redundant mathematical structure in order to extract the physically relevant content of the theory, as the invariant content (Giulini, 2007).

Einstein's notorious "hole argument", Hilbert's version of the argument in terms of Cauchy initial conditions (Stachel, 1988/1992), are precisely the consequence of the existence of mathematical degrees of freedom that do not have any correspondence in physical reality. The covariance of Einstein's equations leave undetermined the evolution of four out of the ten components of $g_{\mu \nu}$. Instead of being a trivial consequence of the application of the "absolute differential calculus" as in "Kretschmann objection", such a "redundancy" in mathematical formalism appears rather to be one of the main philosophical issues raised by General Relativity (Norton, 2003).

Historically, this problem was "rediscovered" in the late 1950's by Peter Bergmann (Bergmann, 1956, 1961b; Bergmann and Komar, 1960; Bergmann, 1961a), Einstein's assistant in Princeton, who was led to re-discuss the question of what is observable in General Relativity. From this vantage point, the Logical Empiricists' idea that the metric is not an "observable", wheres the neighborhood relations encoded in the coordinate system are, appears then utterly inadequate to grasp the relevant philosophical issue; General Relativity shows rather, that because of "the mathematical ambiguity of the coordinate system" (Bergmann, 1956, 491), the values of the metric at a particular world-point are not an observable (Bergmann, 1961b, 1968, §27).

While Reichenbach still in his last writings insisted that the main philosophical issue of Einstein's general theory was the existence of a "class of equivalent descriptions" (Reichenbach, 1953, 133) of different physical situations; after Reichenbach's death it began to become clear that the real problem, as Bergmann put it, is then in 
General Relativity, there is an "equivalence class of solutions" that describes "same physical situation" (Bergmann, 1961b). Only the equivalence class is physically real.

\section{REFERENCES}

Bergmann, P. G. [1956]: 'Fifty Years of Relativity', Science, 123, 3195, pp. 487-494

Bergmann, P. G. [1961a]: 'Gauge-Invariant' Variables in General Relativity', Phys. Rev., 124, pp. $274-278$

Bergmann, P. G. [1961b]: 'Observables in General Relativity', Reviews of Modern Physics, 33, 4, pp. $510-514$

Bergmann, P. G. [1968]: The Riddle of Gravitation, New York: Scribner

Bergmann, P. G. and Komar, A. B. [1960]: 'Poisson Brackets Between Locally Defined Observables in General Relativity', Phys. Rev. Lett., 4, pp. 432-433

Bianchi, L. [1894]: Lezioni di geometria differenziale, Pisa: Spoerri

Bianchi, L. [1902]: 'Relazione sul concorso al Premio Reale, del 1901, per la Matematica', Atti della R. Accademia del Licei, 5, 2, pp. 142-151

Bottazzini, U. [1999]: 'Ricci and Levi-Civita: from Differential invariants to General relativity', in J. Gray (ed), The Symbolic Universe, Oxford/New York: Oxford University Press, pp. 240-257

Butzer, P. [1981]: 'An Outline of the Life and Work of E. B. Christoffel (1829-1900)', Historia Mathematica, 8, 3, pp. 243-276

Carnap, R. [1922]: Der Raum. Ein Beitrag zur Wissenschaftslehre, Berlin: Reuther \& Reichard

Carnap, R. [1923]: 'Über die Aufgabe der Physik und die Anwendung des Grundsatzes der Einfachstheit', Kant-Studien, 28, pp. 90-107

Carnap, R. [1925]: 'Über die Abhängigkeit der Eigenschaften des Raumes von denen der Zeit', KantStudien, 30, p. 331-345

Cartan, E. [1923]: 'Sur les variétés à connexion affine et la théorie de la relativité généralisée (première partie).', Annales scientifiques de l'École Normale Supérieure, 40, pp. 325-412

Cartan, E. [1924a]: 'La théorie des groupes et les recherches récentes en géométrie différentielle', in Proceedings International Mathematical Congress Toronto, Toronto: The University of Toronto Press, vol. 1

Cartan, E. [1924b]: 'Sur les variétés à connexion affine, et la théorie de la relativité généralisée (première partie) (Suite)', Annales scientifiques de l'École Normale Supérieure, 41, pp. 1-25

Cartan, E. [1925]: 'Sur les variétés à connexion affine et la théorie de la relativité généralisée, Part II,', Ann. Ec. Norm., 42, pp. 17-88

Cartan, E. [1931]: 'Geometrie euclidienne et geometrie riemannienne', Scientia (Milano), 42, pp. 393402

Cattani, C. and De Maria, M. [1989]: 'The 1915 Epistolary Controversy between A. Einstein and T. LeviCivita', in D. Howard and J. Stachel (eds), Einstein and the History of General Relativity, New York: Birkhäuser

Christoffel, E. B. [1869]: 'Ueber die Transformation der homogenen Differentialausdrücke zweiten Grades.', Journal für die reine und angewandte Mathematik, 70, pp. 46-70, now in Christoffel, 1910, vol I, 352-377, 378-382

Christoffel, E. B. [1910]: Gesammelte mathematische Abhandlungen, vol. L. Maurer, Leipzig: Teubner

Clifford, W. K. [1876]: 'On the Space-Theory of Matter', Proceedings of the Cambridge philosophical society (1864-1876), 2, pp. 157-158, communications made to the Society, February 21, 1870

Coffa, A. J. [1979]: 'Elective Affinities: Weyl and Reichenbach', in W. C. Salmon (ed), Hans Reichenbach: Logical Empirist, Dordrecht, Boston, London: Kluwer

Coffa, A. J. [1991]: The Semantic Tradition from Kant to Carnap: To the Vienna Station, Cambridge: Cambridge University Press

Corry, L. [2003]: David Hilbert and the Axiomatization of Physics (1898-1918): from Grundlagen der Geometrie to Grundlagen der Physik, Dordrecht: Kluwer

CPAE: Einstein, A. [1996-]: The Collected Papers of Albert Einstein, Princeton: Princeton University Press

Dell'Aglio, L. [1996]: 'On the Genesis of the Concept of Covariant Differentiation', Revue d'histoire des mathématiques, 2, pp. 215-264

Dickson, M. and Domski, M. (eds) [2010]: Discourse on a New Method: Reinvigorating the Marriage of History and Philosophy of Science, Chicago: Open Court

DiSalle, R. [2006]: Understanding Space-Time: The Philosophical Development of Physics from Newton to Einstein, Cambridge, UK ; New York: Cambridge University Press

Earman, J. and Glymour, C. [1978]: 'Lost in the Tensors: Einstein's Struggles with Covariance Principles 1912-1916', Studies In History and Philosophy of Science Part A, 9, 4, pp. $251-278$

Eddington, A. S. [1920]: Space, Time and Gravitation: an Outline of the General Relativity Theory, Cambridge: University Press

Eddington, A. S. [1923]: The Mathematical Theory of Relativity, Cambridge: University Press

Ehlers, J. [1981]: 'Christoffel's Work on the Equivalence Problem for Riemannian Spaces and Its Importance for Modem Field Theories or Physics', in F. Butzer, P.L.; Fehér (ed), E. B. Christoffel: The Influence of his Work on Mathematics and the Physical Sciences, Basel: Birkhäuser

Einstein, A. [1907]: 'Relativitätsprinzip und die aus demselben gezogenen Folgerungen', Jahrbuch der Radioaktivität, 4, p. 411-462, now in CPAE 2, Doc. 47 
Einstein, A. [1914]: 'Die formale Grundlage der allgemeinen Relativitätstheorie', Preussische Akademie der Wissenschaften, Sitzungsberichte, -, p. 1030-1085, now in CPAE 6, Doc. 9

Einstein, A. [1915a]: 'Erklärung der Perihelbewegung des Merkur aus der allgemeinen Relativitätstheorie', Preussische Akademie der Wissenschaften, Sitzungsberichte, -, p. 831-839, now in CPAE 6, Doc. 24

Einstein, A. [1915b]: 'Feldgleichungen der Gravitation', Preussische Akademie der Wissenschaften, Sitzungsberichte, -, p. 844-847, now in CPAE 6, Doc. 25

Einstein, A. [1915c]: 'Grundgedanken der allgemeinen Relativitätstheorie und Anwendung dieser Theorie in der Astronomie', Preussische Akademie der Wissenschaften, Sitzungsberichte, -, p. 315

Einstein, A. [1915d]: 'Zur allgemeinen Relativitätstheorie', Preussische Akademie der Wissenschaften, Sitzungsberichte, -, pp. 778-786, 799-801, now in CPAE 6, Doc. 21 and 22

Einstein, A. [1916a]: 'Über Fr. Kottlers Abhandlung: Einsteins Äquivalenzhypothese und die Gravitation', Annalen der Physik, 51, p. 239, now in CPAE 6, Doc. 40

Einstein, A. [1916b]: 'Die Grundlage der allgemeinen Relativitätstheorie', Annalen der Physik, 49, pp. 769-822, now in CPAE 6, Doc 30

Einstein, A. [1917]: Über die spezielle und die allgemeine Relativitaetstheorie (gemeinverständlich), Braunschweig: Vieweg, now in CPAE 6, Doc. 42

Einstein, A. [1918a]: 'Dialog über Einwände gegen die Relativitätstheorie', Naturwissenschaften, 6, p. 697-702, now in CPAE 7, Doc. 13

Einstein, A. [1918b]: 'Nachtrag zu H. Weyl, Gravitation und Elektrizität', Sitzungsberichte der Preussischen Akademie der Wissenschaften, -, pp. 478-80

Einstein, A. [1918c]: 'Prinzipielles zur allgemeinen Relativitätstheorie', Annalen der Physik, 55, pp. 241-244, now in CPAE 7, Doc. 4

Einstein, A. [1921a]: Geometrie und Erfahrung. Erweiterte Fassung des Festvortrages gehalten an der Preussischen Akademie der Wissenschaften zu Berlin am 2\%. Januar 1921, Springer, Berlin, now in CPAE 7, Doc. 52

Einstein, A. [1921b]: Vier Vorlesungen über Relativitätstheorie, gehalten im Mai 1921, an der Universität Princeton, Braunschweig: Vieweg, now in CPAE 7, Doc. 71

Einstein, A. [1922]: 'Vorwort des Autors zur Tschechischen Ausgabe', Written in 1922 for the Czech edition of Einstein, 1917

Einstein, A. [1925]: 'Nichteuklidische Geometrie und Physik', Die neue Rundschau, 36, pp. 16-20

Einstein, A. [1952]: Über die spezielle und die allgemeine Relativitaetstheorie (gemeinverständlich), Braunschweig: Vieweg, 16 ed.

Einstein, A. and Grossmann, M. [1913]: 'Entwurf einer verallgemeinerten Relativitätstheorie und eine Theorie der Gravitation. I. Physikalischer Teil von A. Einstein II. Mathematischer Teil von M. Grossmann', Zeitschrift für Mathematik und Physik, 62, pp. 225-244, 245-261, now in CPAE 4, 13

Einstein, A. a. a. [1972]: Correspondance 1903-1955: Trad. notes et introd. de Pierre Speziali, Paris: Hermann

Farwell, R. [1990]: 'The Missing Link: Riemann's 'Commentatio,' Differential Geometry and Tensor Analysis', Historia Mathematica, 17, pp. 223-255

Fogel, B. [2008]: Epistemology of a Theory of Everything. Weyl, Einstein, and the Unification of Physics, Notre Dame: University of Notre Dame, Diss.

Friedman, M. [1983]: Foundations of Space-Time Theories: Relativistic Physics and Philosophy of Science, Princeton: Princeton University Press

Friedman, M. [1995]: 'Poincaré's Conventionalism and the Logical Positivists', Foundations of Science, 2, pp. 299-314, now in [Friedman 1999]

Friedman, M. [1999]: Reconsidering Logical Positivism, New York: Cambridge University Press

Friedman, M. [2002]: 'Geometry as a Branch of Physics. Background and Context for Eisnstein's 'Geometry and Experience", in D. Malament (ed), Reading Natural Philosophy, Chicago-La Salle (Ill.): Open Court

Friedman, M. [2008]: 'Space, Time, and Geometry: Einstein and Logical Empiricism', in P. L. Galison, G. J. Holton and S. S. Schweber (eds), Einstein for the 21st Century: his Legacy in Science, Art, and Modern Culture, Princeton: Princeton University Press, pp. 205-216

Gauss, C. F. [1827]: Disquisitiones generales circa superficies curvas, Gottingae

Giulini, D. [2001]: 'Das Problem der Trägheit', MPIGW-Preprint, 190

Giulini, D. [2007]: 'Remarks on the Notions of General Covariance and Background Independence', Lecture Notes in Physics, 721, pp. 105-120

Hawkins, T. [1980]: 'Non-Euclidean Geometry and Weierstrassian Mathematics: The Background to Killing's Work on Lie Algebras', Historia Mathematica, 7, 3, pp. $289-342$

Hawkins, T. [2000]: Emergence of the Theory of Lie groups: an Essay in the History of Mathematics, 1869 - 1926, New York: Springer

Helmholtz, H. [1868]: 'Über die Tatsachen, die der Geometrie zu Grunde liegen', Nachrichten von der Königl. Gesellschaft der Wissenschaften und der Universität zu Göttingen, 9, pp. 193-221

Helmholtz, H. [1870/1883]: 'Über Ursprung und Bedeutung der Geometrischen Axiome', in Vorträge und Reden, Braunschweig; Wiesbaden: Vieweg, pp. 1-31

Helmholtz, H. [1878]: 'The Origin and Meaning of Geometrical Axioms II', Mind, 3, pp. 212-225

Helmholtz, H. [1879]: Die Thatsachen in der Wahrnehmung: Rede gehalten zur Stiftungsfeier der Friedrich-Wilhelms-Universität zu Berlin am 3. August 1878, Berlin: A. Hirschwald

Helmholtz, H. [1921]: Schriften zur Erkenntnistheorie, Berlin: Springer

Herbert, D. [1991]: Die Entstehung des Tensorkalküls : von den Anfängen in der Elastizitätstheorie bis zur Verwendung in der Baustatik, Stuttgart: Steiner 
Hessenberg, G. [1917/18]: 'Vektorielle Begründung der Differentialgeometrie', Mathematische Annalen, 78, pp. $187-217$

Howard, D. [1984]: 'Realism and Conventionalism in Einstein's Philosophy of Science: The EinsteinSchlick Correspondence.', Philosophia naturalis, 21, pp. 618-29

Howard, D. [1990]: 'Einstein and Duhem', Synthese, 83, pp. 363-384

Howard, D. [1994]: 'Einstein, Kant and the Origins of Logical Empiricism', in W. C. Salmon and G. Wolters (eds), Logic, Language, and the Structure of Scientific theories: Proceedings of the Carnap-Reichenbach Centennial, University of Konstanz, 21-24 May 1991, Pittsburgh, Pa.; Konstanz: University of Pittsburgh Press; Universitätsverlag Konstanz

Howard, D. [2005]: 'Einstein's Philosophy of Science', in E. N. Zalta (ed), The Stanford Encyclopedia of Philosophy (Spring 2004 Edition), -

Janssen, M. [2005]: 'Of Pots and Holes: Einstein's Bumpy Road to General Relativity', Annalen der Physik, 14; Supplement, pp. 58-85

Janssen, M. [2008]: 'No Success like Failure ...': Einstein's Quest for General Relativity, 1907-1920',

Janssen, M. [2011]: 'The Twins and the Bucket: How Einstein made Gravity rather than Motion Relative in General Relativity',

Janssen, M. and Renn, J. [2007]: 'Untying the Knot: how Einstein Found his way Back to Field Equations Discarded in the Zurich Notebook', in M. Janssen, J. D. Norton, J. Renn, T. Sauer and J. Stachel (eds), The Genesis of General Relativity, Springer Netherlands, vol. 250 of Boston Studies in the Philosophy of Science, pp. 839-925

Koenigsberger, L. . [1906]: Hermann von Helmholtz, Oxford: Clarendon Press

Kottler, F. [1916]: 'Über Einsteins Äquivalenzhypothese und die Gravitation', Annalen der Physik, 355, 16, pp. 955-972

Kretschmann, E. [1917]: 'Über den physikalischen Sinn der Relativitätspostulate. A. Einsteins neue und seine ursprüngliche Relativitätstheorie', Annalen der Physik, 53, pp. 575-614

Lehmkuhl, D. [2008]: 'Is Spacetime a Gravitational Field?', in D. Dieks (ed), The Ontology of Spacetime II, Elsevier Science

Levi-Civita, M. [1916]: 'Nozione di parallelismo in una varietà qualunque e conseguente specificazione geometrica della curvatura riemanniana', Rendiconti del Circolo Matematico di Palermo (1884 1940), 42, pp. 173-204, 10.1007/BF03014898

Levi-Civita, T. . [1927]: The Absolute Differential Calculus (Calculus of Tensors), London/Glasgow: Blackie \& Son

Levi-Civita, T. [1925]: Lezioni di calcolo differenziale assoluto, Roma: Stock

Levi-Civita, T. and Ricci-Curbastro, G. [1900]: 'Méthodes de calcul différentiel absolu et leurs applications', Mathematische Annalen, 54, pp. 125-201, now in Ricci-Curbarstro 1956-57, I,

Libois, P. [1957]: 'Quelques applications des idees de Riemann sur l'espace', in J. Naas and K. Schröder (eds), Der Begriff des Raumes in der Geometrie: Bericht von der Riemann-Tagung des Forschungsinstituts für Mathematik, Berlin: Akademie-Verlag, pp. 194-201

Lie, S. [1886]: 'Bemerkungen zu v. Helmholtz Arbeit über die Tatsachen, welche der Geometrie zugrunde liegen', Berichte über die Verhandlungen der Kgl. Sächsischen Gesellschaft der Wissenschaften zu Leipzig, 38, pp. 337-342

Lipschitz, R. [1869]: 'Untersuchungen in Betreff der ganzen homogenen Functionen von n Differentialen', Journal für die reine und angewandte Mathematik, 70, pp. 71-102

Maltese, G. [1991]: 'The Rejection of the Ricci Tensor in Einstein's First Tensorial Theory of Gravitation', Archive for History of Exact Sciences, 41, 4, pp. 363-381

Mehra, J. [1974]: Einstein, Hilbert, and the Theory of Gravitation : Historical Origins of General Relativity Theory, Dordrecht [et al.]: Reidel

Nerlich, G. [1994]: What Spacetime Explains: Metaphysical Essays on Space and Time, Cambridge [et al.]: Cambridge Univ. Press

Norton, J. [1985]: 'What was Einstein's Principle of Equivalence?', Studies in History and Philosophy of Science Part A, 16, 3, pp. 203-246

Norton, J. D. [1984]: 'How Einstein found his Field Equations: 1912-1915', Historical Studies in the Physical Sciences, 14, pp. 253-316

Norton, J. D. [1987]: 'Einstein, the Hole Argument and the Reality of Space', in J. . Forge (ed), Measurement, Realism, and Objectivity : Essays on Measurement in the Social and Physical sciences, Dordrecht/Boston/London: Kluwer

Norton, J. D. [1994]: 'Why Geometry is not Conventional: The Verdict of Covariance Principles', in U. Majer and H.-J. Schmidt (eds), Semantical aspects of spacetime theories, Mannheim [at al.]: BI-Wissenschaftsverl., pp. 159-167

Norton, J. D. [2002]: 'Einstein's Triumph over the Spacetime Coordinate System. A Paper presented in Honor of Roberto Torretti', Dialogos, 79, 2002, pp. 253-262

Norton, J. D. [2003]: 'General Covariance, Gauge Theories, and the Kretschmann Objection', in K. Brading and E. Castellani (eds), Symmetries in Physics. Philosophical Reflections, Cambridge: Cambridge University Press, pp. 110-123

Ohanian, H. C. [1977]: 'What is the Principle of Equivalence?', American Journal of Physics, 45, p. 903

Pais, A. [1982]: Subtle is the Lord: The Science and the Life of Albert Einstein, New York: Oxford University Press

Parrini, P. [2005]: L'empirismo logico. Aspetti storici e prospettive teoriche, Roma: Carocci

Pauli, W. [1921]: 'Relativitätstheorie', in Enzyklopädie der mathematischen Wissenschaften, Teubner, vol. 5, pp. 539-775 
Pesic, P. [2007]: Beyond geometry. Classic Papers from Riemann to Einstein, Mineola, N.Y.: Dover Publications

Poincaré, H. [1887]: 'Sur les hypothéses fondamentales de la géométrie', Bulletin de la Société mathématique de France, 15, pp. 203-216

Poincaré, H. [1891]: 'Les géométries non euclidiennes', Revue générale des sciences pures et appliquées, 2, pp. $769-774$

Poincaré, H. [1882]: 'Théorie des groupes fuchsiens', Acta Mathematica, 1, pp. 1-62

Portnoy, E. [1982]: 'Riemann's Contribution to Differential Geometry', Historia Mathematica, 9, 1, pp. $1-18$

Redhead, M. [2001]: 'The Intelligibility of the Universe', in A. O'Hear (ed), Philosophy at the New Millennium, Cambridge: Cambridge University Press

Reich, K. [1992]: 'Levi-Civitasche Parallelverschiebung, affiner Zusammenhang, Übertragungsprinzip: 1916/17-1922/23', Archiv für Geschichte der Philosophie, 44, 1, pp. 77-105

Reich, K. [1994]: Die Entwicklung des Tensorkalküls: vom absoluten Differentialkalkül zur Relativitätstheorie, Berlin: Birkhäuser

Reichenbach, H. [1920a]: 'Die Einsteinsche Raumlehre', Die Umschau, 24, pp. 402-405

Reichenbach, H. [1920b]: Relativitätstheorie und Erkenntnis apriori, Springer

Reichenbach, H. [1921]: 'Der gegenwärtige Stand der Relativitätsdiskussion. Eine kritische Untersuchung', Logos, 22, 10, pp. 316-378

Reichenbach, H. [1922]: 'La signification philosophique de la théorie de la relativité', Revue philosophique de la France et de l'Étranger, 93, pp. 5-61

Reichenbach, H. [1924]: Axiomatik der relativistischen Raum-Zeit-Lehre, Vieweg: Braunschweig

Reichenbach, H. [1927]: Von Kopernikus bis Einstein. Der Wandel unseres Weltbildes, Berlin: Ullstein

Reichenbach, H. [1928]: Philosophie der Raum-Zeit-Lehre, Berlin and Leipzig: Walter de Gruyter, now in Reichenbach 1977, vol II

Reichenbach, H. [1929a]: 'Ziele und Wege der physikalischen Erkenntnis', in Handbuch der Physik, vol. 4: Allgemeine Grundlagen der Physik, Berlin: Springer, pp. 1-80

Reichenbach, H. [1929b]: 'Zur Einordnung des neuen Einsteinschen Ansatzes über Gravitation und Elektrizität', Zeitschrift für Physik, 59, nos. 9-10, pp. 683-689

Reichenbach, H. [1938]: Experience and prediction: an analysis of the foundations and the structure of knowledge, Chicago [u.a.]: Univ. of Chicago Press

Reichenbach, H. [1953]: The Rise of Scientific Philosophy, Berkely/Los Angeles/London: University of California Press

Reichenbach, H. [1958]: The Philosophy of Space and Time, New York: Dover Publ.

Reichenbach, H. [1977]: Gesammelte Werke in 9 Bänden, Braunschweig; Wiesbaden: Vieweg

Reichenbächer, E. [1923]: 'Träge, Schwere und felderzeugende Masse', Zeitschrift für Physik, 15, pp. $276-279$

Ricci-Curbastro, G. [1883]: 'Principii di una teoria delle forme differenziali quadratiche', Annali di Matematica Pura ed Applicata, 12, pp. 135-167, now in Ricci-Curbarstro 1956-57, I,

Ricci-Curbastro, G. [1886]: 'Sui parametri e gli invarianti delle forme quadratiche differenziali', Annali di Matematica Pura ed Applicata (1867 - 1897), 14, pp. 1-11, now in Ricci-Curbarstro 1956-57, I,

Ricci-Curbastro, G. [1888]: 'Delle Derivazioni covarianti e controvarianti e del loro uso nella analisi applicata', in Studi editi dalla Università di Padova a commemorare l'ottavo centenario della Università di Bologna, Padova: Tip. del Seminario, vol. 3, pp. 3-23

Ricci-Curbastro, G. [1889]: 'Sopra certi sistemi di funzioni', Atti Accad. Lincei, 4/6, pp. 112-118, now in Ricci-Curbarstro 1956-57, I,

Ricci-Curbastro, G. [1892]: 'Le calcul différentiel absolu', Bulletin des sciences mathématiques, 16, pp. $167-189$

Ricci-Curbastro, G. [1893]: 'Di alcune applicazioni del Calcolo differenziale assoluto alla teoria delle forme differenziali quadratiche binarie e dei sistemi a due variabili', Atti dell'Istituto Veneto di scienze, lettere ed arti, 7, 4, pp. 1336-1364, now in Ricci-Curbarstro 1956-57, I, 311-335.

Ricci-Curbastro, G. [1956-57]: Opere, Roma: Cremonese

Riemann, B. [1854/1868]: 'Ueber die Hypothesen, welche der Geometrie zu Grunde liegen (Aus dem Nachlass des Verfassers mitgetheilt durch R. Dedekind)', Abhandlungen der Königlichen Gesellschaft der Wissenschaften zu Göttingen, 13, pp. 132-152

Riemann, B. [1861/1876]: 'Commentatio mathematica, qua respondere tentatur quaestioni ab Illma Academia Parisiensi propositae', in Bernhard Riemann's Gesammelte Mathematische Werke, Weber, Heinrich, pp. 391-404

Riemann, B. [1873]: 'On the Hypotheses which lie at the Bases of Geometry (translated by', Nature, 8, pp. 14-17; 36-37, translated by Clifford, William Kingdom

Roth, L. [1942]: 'Obituaries. Prof. Tullio Levi-Civita', Nature, 149, p. 266.

Ryckman, T. [1992]: '(P)oint-(C)oincidence Thinking. The Ironical Attachment of Logical Empiricism to General Relativity', Studies in History and Philosophy of Modern Physics, 23, 3, pp. 471-497

Ryckman, T. [2007]: 'Logical Empiricism and Philosophy of Physics', in A. W. Richardson and T. Uebel (eds), The Cambridge Companion to Logical Empiricism, Cambridge [et al.]: Cambridge University Press, 1. publ. ed., pp. XI, 430 S.

Ryckman, T. [2008]: 'Early Philosophical Interpretations of General Relativity', in idem, The Stanford Encyclopedia of Philosophy: Fall 2008 Edition, ed. by E. N. Zalta, plato.stanford.edu/entries/genrel-early/

Rynasiewicz, R. [1999]: 'Kretschmann's Analysis of Covariance and Relativity Principles', in H. Goenner (ed), The Expanding Worlds of General Relativity, Basel: Birkhäuser, pp. 431-462 
Sauer, T. [2005]: 'Albert Einstein's 1916 Review Article on General Relativity', in I. Grattan-Guinness and R. Cooke (eds), Landmark Writings in Western mathematics 1640-1940, Elsevier, pp. 802-822 Schlick, M. [1917a]: 'Raum und Zeit in der gegenwärtigen Physik. Zur Einführung in das Verständnis der allgemeinen Relativitätstheorie', Die Naturwissenschaften, 5, pp. 161-167; 177-186

Schlick, M. [1917b]: Raum und Zeit in der gegenwärtigen Physik. Zur Einführung in das Verständnis der allgemeinen Relativitätstheorie, Berlin: Spr

Schlick, M. [1922/2006]: 'Raum und Zeit in der gegenwärtigen Physik', in M. Neuber and O. Engler (eds), Gesamtausgabe, New York/Berlin: Springer, vol. I,2

Schlick, M. and Reichenbach, H. [1920-22]: 'Correspondence 1920-22',

Scholz, E. [1982]: 'Riemanns frühe Notizen zum Mannigfaltigkeitsbegriff und zu den Grundlagen der Geometrie', Archive for History of Exact Sciences, 27, 3, pp. 213-232

Scholz, E. [1992]: 'Riemann's Vision of a New Approach to Geometry', in L. Boi, D. Flament and J.M. Salanskis (eds), 1830-1930: A Century of Geometry. Epistemology, History and Mathematics, Springer, New York/Berlin, pp. 22-34

Schouten, J. A. [1919]: 'Die direkte Analysis zur neueren Relativitätstheorie', Verhandlingen Kgl. Akademie der Wetenschappen Amsterdam, 12, 6

Stachel, J. [1980/2002]: 'Einstein's Search for General Covariance, 1912-1915', in Einstein from "B" to " $Z$ ". Birkhäuser, Boston, pp. 301-337, the paper was first read at the Ninth International Conference on General Relativity and Gravitation, Jena in 1980

Stachel, J. [1986]: 'What can a Physicist Learn from the Discovery of General Relativity?', in R. Ruffini (ed), Proceedings of the Fourth Marcel Grossmann Meeting on Recent Developments in General Relativity, Amsterdam: North-Holland, pp. 1857-62

Stachel, J. [1988/1992]: 'The Cauchy Problem in General Relativity-The Early Years', in J. H. Eisenstaedt (ed), Studies in the History of General Relativity: Based on the Proceedings of the 2nd International Conference on the History of General Relativity, Luminy, France, 1988, Boston [et al.]: Birkhäuser, pp. 407-418

Stachel, J. [1989]: 'The Rigidly Rotating Disk as the 'Missing Link in the History of General Relativity', in D. Howard and J. Stachel (eds), Einstein and the History of General Relativity (Einstein Studies, Vol. 1), Boston: Birkhäuser, p. 48-62

Stachel, J. [2007]: 'The Story of Newstein or: Is Gravity Just Another Pretty Force?', in M. Janssen, J. D. Norton, J. Renn, T. Sauer and J. Stachel (eds), The Genesis of General Relativity, Springer Netherlands, vol. 250 of Boston Studies in the Philosophy of Science, pp. 1962-2000

Struik, D. J. [1989]: 'Schouten, Levi-Civita and the Emergence of Tensor Calculus', in D. E. Rowe and J. McCleary (eds), History of Modern Mathematics, Boston: Academic Press, vol. 2: Institutions and Applications.

Struik, D. J. [1993]: 'From Riemann to Ricci : the Origins of the Tensor Calculus', in H. M. H. Srivastava (ed), Analysis, Geometry and Groups : a Riemann Legacy Volume, Palm Harbor, Fla.: Hadronic Press

Synge, J. [1960]: Relativity: the General Theory, Amsterdam; New York: North-Holland Pub. Co.; Interscience Publishers

Synge, J. L. [1970]: Talking about Relativity, Amsterdam [u.a.]: North-Holland

Tonolo, A. [1961]: 'Sulle origini del Calcolo di Ricci', Annali di Matematica Pura ed Applicata, 53, pp. $189-207$

Torretti, R. [1983]: Relativity and Geometry, Oxford: Pergamon Press

Toscano, F. [2001]: 'Luigi Bianchi, Gregorio Ricci Curbastro e la scoperta delle identità di Bianchi', in Atti del XX Congresso Nazionale di Storia della Fisica e dell'Astronomia, Napoli: CUEN, pp. $353-370$

Weyl, H. [1918a]: 'Gravitation und Elektrizität', Sitzungsberichte der preuß. Akad. Berlin, Math. Kl., - , pp. 465-480, now in WGA II, pp. 29-42

Weyl, H. [1918b]: Raum, Zeit, Materie: Vorlesungen über allgemeine Relativitätstheorie, Berlin: Springer

Weyl, H. [1918s]: 'Reine Infinitesimalgeometrie', Mathematische Zeitschrift, 2, pp. 384-411, now in WGA II, Doc. 30

Weyl, H. [1919]: Raum, Zeit, Materie: Vorlesungen über allgemeine Relativitätstheorie [2nd ed.], Berlin: Springer

Weyl, H. [1920]: 'Die Diskussion über die Relativitätstheorie', Die Umschau, 24, pp. 609-611

WGA: Weyl, H. [1968]: Gesammelte Abhandlungen, Berlin: Springer

Zund, J. [1983]: 'Some Comments on Riemann's Contributions to Differential Geometry', Historia Mathematica, 10, pp. 84-89 